Article

\title{
The Impact of Surrogate Models on the Multi-Objective Optimization of Pump-As-Turbine (PAT)
}

\author{
Stephen Ntiri Asomani ${ }^{1}$, Jianping Yuan ${ }^{1,2, *}$, Longyan Wang ${ }^{1,3, *}$, Desmond Appiah ${ }^{1}$ \\ and Kofi Asamoah Adu-Poku ${ }^{1}$ \\ 1 National Research Center of Pumps, Jiangsu University, Zhenjiang 212013, China; \\ 5103170318@stmail.ujs.edu.cn (S.N.A.); bembo88donk@ujs.edu.cn (D.A.); \\ kofi.adu-poku@uenr.edu.gh (K.A.A.-P.) \\ 2 Institute of Fluid Engineering Equipment, JITRI, Jiangsu University, Zhenjiang 212013, China \\ 3 School of Chemistry, Physics and Mechanical Engineering, Queensland University of Technology, \\ Brisbane 4001, Australia \\ * Correspondence: yh@ujs.edu.cn (J.Y.); longyan.wang@connect.qut.edu.au (L.W.)
}

Received: 24 February 2020; Accepted: 30 April 2020; Published: 4 May 2020

\begin{abstract}
Pump-as-turbine (PAT) technology permits two operating states-as a pump or turbine, depending on the demand. Nevertheless, designing the geometrical components to suit these operating states has been an unending design issue, because of the multi-conditions for the PAT technology that must be attained to enhance the hydraulic performance. Also, PAT has been known to have a narrow operating range and operates poorly at off-design conditions, due to the lack of flow control device and poor geometrical designs. Therefore, for the PAT to have a wider operating range and operate effectively at off-design conditions, the geometric parameters need to be optimized. Since it is practically impossible to optimize more than one objective function at the same time, a suitable surrogate model is needed to mimic the objective functions for it to be solvable. In this study, the Latin hypercube sampling method was used to obtain the objective function values, the Adaptive Neuro-Fuzzy Inference System (ANFIS), Artificial Neural Network (ANN) and Generalized Regression Neural Network (GRNN) were used as surrogate models to approximate the objective functions in the design space. Then, a suitable surrogate model was chosen for the optimization. The Pareto-optimal solutions were obtained by using the Pareto-based genetic algorithm (PBGA). To evaluate the results of the optimization, three representative Pareto-optimal points were selected and analyzed. Compared to the baseline model, the Pareto-optimal points showed a great improvement in the objective functions. After optimization, the geometry of the impeller was redesigned to suit the operating conditions of PAT. The findings show that the efficiencies of the optimized design variables of PAT were enhanced by $23.7 \%, 11.5 \%$, and $10.4 \%$ at part load, design point, and under overload flow conditions, respectively. Moreover, the results also indicated that the chosen design variables $\left(b_{2}, \beta_{2}, \beta_{1}\right.$, and $\left.z\right)$ had a substantial impact on the objective functions, justifying the feasibility of the optimization method employed in this study.
\end{abstract}

Keywords: pump as turbine; optimization; multi-objective; General Regression Neural Network; Adaptive Neuro-Fuzzy Inference System

\section{Introduction}

The generation of electricity presents numerous problems, which have been addressed through the years by several methods to reduce its operational costs and environmental effects. The production of electricity through fossil fuels poses high effects on the ozone layer by depletion. This depletion of the ozone layer causes global warming, which is detrimental to aquatic life and ecosystems at large [1]. 
Renewable energy such as the solar power, biofuels, and hydropower can be a suitable solution for ecological problems. However, of all the green energy sources, hydropower provides a suitable means of energy production. The flexibility and storage capability of hydropower plants render them more economical, which encourages power supply and market stability. In addition, hydropower helps to reduce carbon emissions, improving air quality. Hydropower is energy generated through hydraulic turbines from water sources like the ocean, rivers, and waterfalls. Hydraulic turbines are known for high energy efficiency; however, their implementation is reasonably unattractive due to the high purchase for small hydropower [2]. Pump-as-turbine (PAT), alternatively, is a simple hydraulic machine that can be used to generate electricity using hydropower systems. The PAT can be used simultaneously as a pump and turbine without any major geometrical parameter change [3].

The use of pump-as-turbine (PAT) is described by Ramos and Borga [4] as the best substitute for utilizing the excess energy available at natural falls, irrigation systems, water supply, wastewater, and rain systems in normal conditions. Steady state conditions were analyzed based on Suter's parameters to show that the pump can be used in industry processes and renewable energy production. In conclusion, PAT technology becomes an innovative solution for the development of electricity, whatever form of motor or alternator. Mankbadi and Mikhail [5] have developed a method for defining the system's key design parameters that can be used to mount the PAT system through irrigation structures. The available low-head energy may be used in irrigation channels to drive a turbine connected to a pump, which can be used to raise water for irrigation or household purposes. It has been concluded that the off-design performance of the system can be managed to achieve a full pumped flow throughout the year. Thus, wherever low head energy is accessible, the PAT technology can be considered.

Ramos et al. [6] investigated the hydraulic system response by using numerical simulations (steady and unsteady) to analyze the results in piping systems between a pressure reducing valve (PRV) and PAT (working as PRV). It was established that it is possible to use PAT as PRV and also to produce electricity with the energy stored in pressured water. It was found that in some situations PAT would work better than PRV, while in others it is recommended that both PAT and PRV be used. A $97 \mathrm{~kW}$ micro hydropower system was designed by Teuteberg [7] in South Africa at Roman Bay Sea Farm. The sea water was pumped into tanks and then supplied through the farm by virtue of gravity. After passing by the farm, the water returns through a single pipe back to the ocean. This water still has enough energy to produce electricity.

The operational specification requirements of PAT (pump and turbine mode), such as the pressure head, rotational speed, and design flow rate, is a tedious and time-consuming part. These design parameters are required to minimize cavitation in order to increase the hydraulic efficiency and energy generation of the PAT operation. Furthermore, by comparing the flow passages of PAT, the pump mode has a diffused flow passage while the turbine mode has a contracted flow passage. The diffused flow channel occurs due to the increase in the cross-sectional area of the volute from the tongue to the volute throat in pump mode. While, the flow passage contracted in turbine mode, owing to a decrease in the sectional-sectional area from the throat to the tongue region. The hydraulic flow losses of the contracted flow channel is supposed to remain smaller than that of the diffused flow channel, but the turbine mode performance in comparison to pump mode is very weak at off-design flow conditions. This negative occurrence has been attributed to the change in flow direction, which sharply increases the hydraulic and frictional losses. These phenomena have also been attributed to the fixed geometric parameters (impeller and volute) and the absence of flow control devices. This implies that the geometrical parameters of the pump do not perfectly suit the turbine mode operation and hence the pump needs to be optimized to improve the performance of the PAT. Thus, this study seeks to optimize the pump's geometrical parameters with good precision so as to improve the performance and thereby broaden the operational range of the turbine mode.

Yang et al. [8] established that, as the pumps were not designed to operate as turbines, the hydraulic losses in the impeller flow zone of the turbine mode operation accounts for more than $50 \%$ of the 
overall hydraulic losses. This is because the pump impellers are designed for forwarding rotation without taking into account the reverse mode operation. This observation also implies that the poor performance of PAT (turbine mode) could be attributed to the geometry of the impeller. Kong [9] subsequently argues that, unlike the conventional turbine which has a guide vane system, during PAT mode the inlet blade angle becomes fixed for the overall operating range, leading to the formation of a vortex. This vortex induces a pressure difference from the inlet and the outlet of the volute to increase the loss of leakage. So, compared with pump mode, the lower efficiency and higher flow instabilities of PAT are usually derived from the poor design of the flow passage and impeller-volute interaction. Extensive studies by Yang et al. [8] on different blade wrap angles under different specific speeds in PAT revealed that, to enhance its performance, there is an optimal wrap angle, which decreases with increasing specific speed. Following the observations of the aforementioned studies, Yang et al. [10] showed that it would be more appropriate if the value of the inlet blade angle falls within the range of 25 and 35 for PAT with a spiral case. Derakhshan et al. [11], also suggested modifying the impeller's geometry by rounding the leading edges of the blades and the face of the shroud or hub to enhance the hydraulic efficiency. The geometry of the impeller blade influences the overall efficiency of the pumps and PAT.

Research has indicated that the traditional method employed by pump manufacturers for pump design and optimization is mainly the trial-and-error strategy, which is expensive and time-consuming [12]. Therefore, for pump performance improvement, the combination of the optimization method with a computational fluid dynamics (CFD) numerical simulation will decrease the developmental cycle and experimental resources. Furthermore, to achieve optimal results, the best combination of geometrical parameters can also be determined. However, the likelihood of conflict existing between different objective functions is high, because it is practically impossible to optimize more than one objective function at the same time. CFD and experimental test methods can be used to achieve such results. Nonetheless, none of those findings obtained from the CFD simulation and test can be used to improve the pump performance. Therefore, the alternative is to use a suitable surrogate model to mimic the output between the design variables and the objective functions. Surrogate modeling is an inferred prediction process of the input and output when an intended outcome cannot be easily determined [13]. Compared to other optimization methods, the surrogate-based model optimization can effectively determine the behavior of the objective functions more efficiently at a cheaper computational cost.

The most frequently used surrogate models are response surface methodology [14], Artificial Neural Network [15], the kriging model [13], Adaptive Neuro-Fuzzy Inference System [16], and Generalized Regression Neural Network [17]. Surrogate simulation approaches have been comprehensively implemented in the multi-objective optimization of turbomachinery and other fields [18-20]. Zhang et al. [21] suggested a multi-objective and multi-operating optimization approach for the design of a multi-phase pump impeller using a combination of CFD, Back-propagation (BP) Neurons Network and NSGA-II (non-dominated sorting genetic algorithm). The authors applied the CFD findings to train neural networks to find objective functions. Safikhani et al. [22] also suggested a multi-objective centrifugal optimization method based on the neural network surrogate model for genetic algorithms and achieving the optimal output using Pareto.

Nourbakhsh et al. [23] applied different algorithms, such as NSGA II, particle swarm optimization (PSO), and neural network (NN), to determine the Pareto frontier of the two conflicting outputs, which are efficiency and the NPSHr (net positive suction head required) of the centrifugal pump. Derakhshan et al. [24] redesigned the Berkesh 32-160 geometry of the pump impellers to improve the hydraulic efficiency using a global optimization approach focused on the ANN and Artificial Bee Colony, along with a validated 3-D Navier-Stokes flow solver. An airfoil wind turbine was developed by Djavareshkian and Latifi [25], using the Genetic Algorithm (GA), CFD, and ANN models as predictive models to minimize compute costs and time. An optimization by Giugni et al. [26] was carried out using a hydraulic solver Genetic Algorithm (GA) supported by the Newton Iterative 
Solver (NITSOL). They studied the process of energy recovery of the water distribution system by coupling and replacing the pressure reduction valve (PRV) with a pump-as-turbine (PAT). Using their experimental data, an Artificial Neural Network (ANN)-based model was put forward by Pandey and Saini [27]. The Matlab NN-toolbox (Neural Network toolbox) software was applied for the study of the PAT. Performance curves were established for the power output and efficiency. The model was used to prepare, validate, and analyze data at the different head and flow rates from 7 to $21 \mathrm{~m}$ and from 24.5 to $50 \mathrm{r} / \mathrm{min}$. Head, flow rate, and power input were taken as input parameters and the PAT output and efficiency were considered to be the target data for the model. To validate the ANN model, a comparison was made between the ANN result and the actual experimental data. The findings indicated that the ANN model was apt for assessing the performance of PAT at different heads and flow rates. Thus, the ANN model could be considered as an appropriate tool for the selection of pump-as-turbine (PAT).

The objective of this study was to find a suitable computationally cost-effective and less complex surrogate model to optimize the performance of PAT (turbine mode) at design and off-design conditions. Although surrogate models have been successfully employed for pump performance optimization, their influence on the prediction of PAT (turbine mode) has not been extensively studied. This research, therefore, suggested an optimization technique incorporating DOE (design of experiment), surrogate models and a Pareto-based genetic algorithm (PBGA), with a numerical simulation to improve the efficiency of PAT and also broaden its operating range at part load, design point, and overload flow condition $\left(0.6 Q_{d}, 1.0 Q_{d}\right.$, and $1.4 Q_{d}$, respectively). The proposed model was then applied to construct a nonlinear function between the objective functions and the design variables at design and off-design flow conditions. Furthermore, the prediction accuracy of different surrogate models, including ANFIS, ANN, and GRNN, were also compared and analyzed. The best efficiency and combination of design variables was achieved with the PBGA.

\section{PAT Model}

\subsection{Centrifugal PAT Model}

A centrifugal PAT was used as the research object. The PAT consisted of an impeller with six blades and a spiral volute. The design flow rate and head were $25 \mathrm{~m}^{3} / \mathrm{h}$ at $Q_{d}$ with a rotational speed of $1450 \mathrm{r} / \mathrm{min}$ and $8.5 \mathrm{~m}$, respectively. The main parameters of the PAT model are shown in Table 1 . Figure 1 shows a sectional view of the impeller and volute in 2D, while Figure 2 also depicts the actual structure of the PAT.

Table 1. Geometrical and operational parameters of the studied PAT.

\begin{tabular}{ccccc}
\hline Description & Parameter & Symbol & Unit & Value \\
\hline Impeller & Outlet diameter & $D_{2}$ & $\mathrm{~mm}$ & 174 \\
& inlet diameter & $\mathrm{Dj}^{\mathrm{j}}$ & $\mathrm{mm}$ & 74 \\
& Outlet width & $b_{2}$ & $\mathrm{~mm}$ & 12 \\
& Blade number & $z$ & - & 6 \\
& Hub diameter & $D_{h}$ & $\mathrm{~mm}$ & 21 \\
& Inlet diameter & $D_{3}$ & $\mathrm{~mm}$ & 184 \\
& Inlet width & $b_{3}$ & $\mathrm{~mm}$ & 20 \\
& Tongue angle & $\theta$ & $\circ$ & 27 \\
Operational Condition & Nominal flow rate & $Q$ & $\mathrm{~m}^{3} / \mathrm{h}$ & 25 \\
& Rotation speed & $n$ & $\mathrm{r} / \mathrm{min}$ & 1450 \\
& Efficiency & $\eta$ & $(\%)$ & 72.1 \\
& Head & $H$ & $\mathrm{~m}$ & 8.5 \\
\hline
\end{tabular}




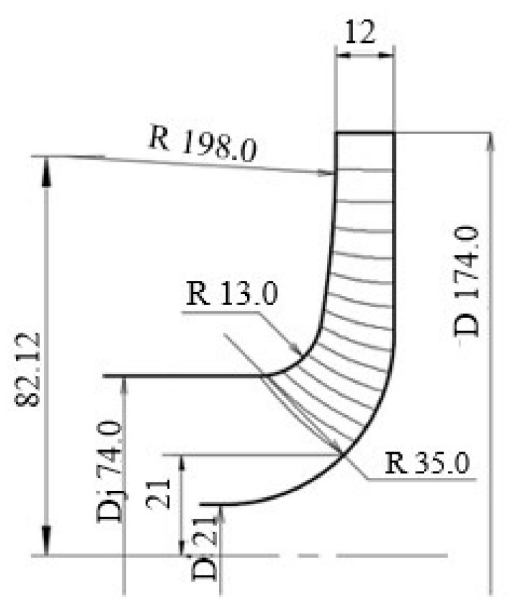

(a)

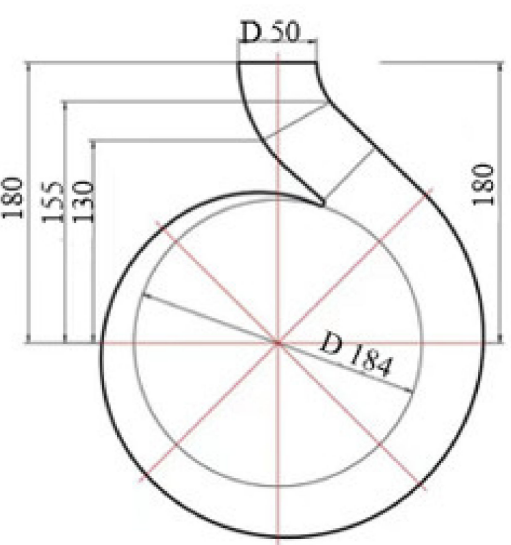

(b)

Figure 1. Sectional view of (a) impeller, (b) volute.

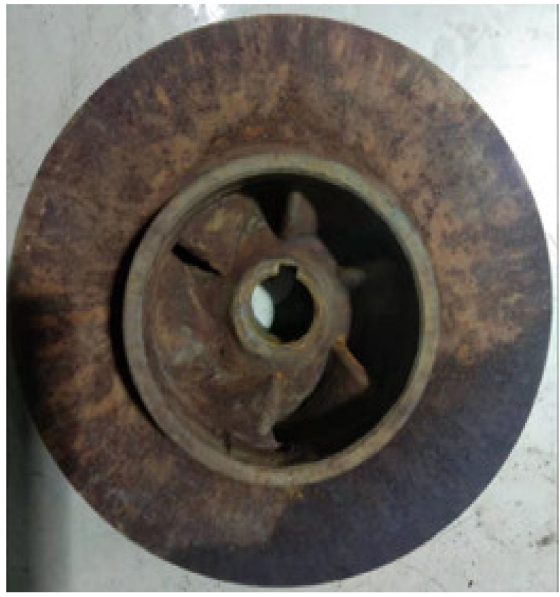

(a)

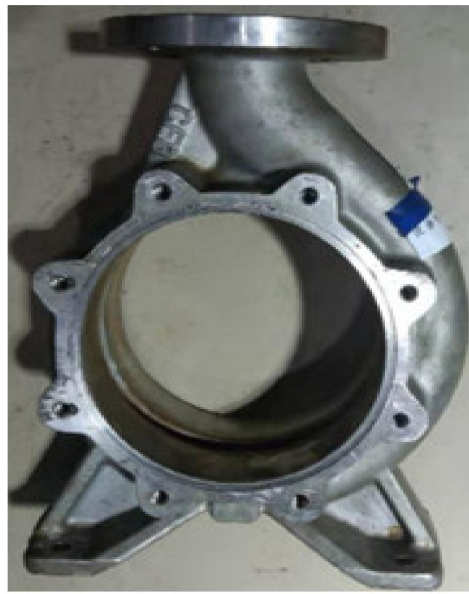

(b)

Figure 2. Pump-as-turbine (PAT) model: (a) impeller, (b) volute.

\subsection{Mesh Generation}

Comparing structured mesh to unstructured mesh, structured mesh may converge quicker than unstructured mesh. Before the numerical simulation, the mesh quality and mesh number are crucial to the optimization process. ANSYS-ICEM was used to generate a structured grid for the computational domain. Figure 3 shows a summary of the computational mesh generated. The grids were defined in the near-wall flow regions and the mesh independence check was performed with the grid numbers [28]. The total number of grids was approximately 2.9 million. 




(a)

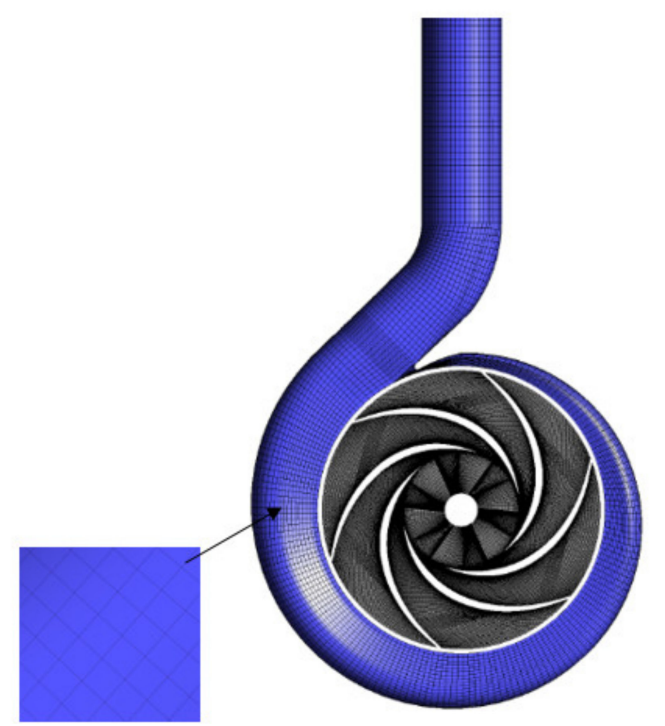

(c)

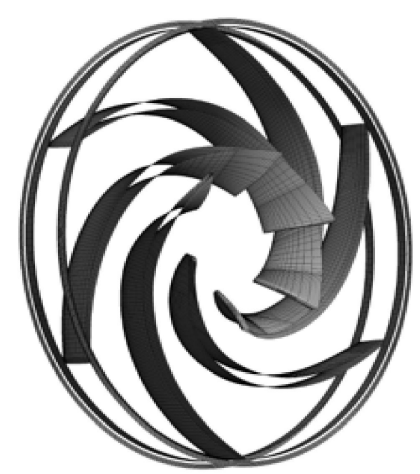

(b)

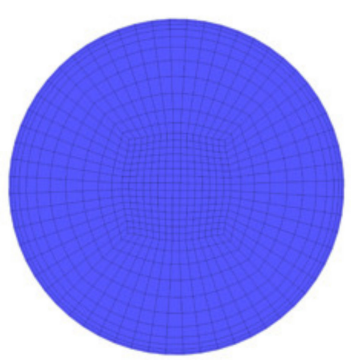

(d)

Figure 3. Computational grid domains. (a) Shroud, (b) impeller blades, (c) volute, (d) inlet.

\subsection{Grid-Sensitivity Analysis}

The grid-sensitivity was performed using the PAT model. The blade exit angles of the hub and shroud is $30^{\circ}$ respectively, with a blade wrapping angle of $140^{\circ}$ and a flow rate of $25 \mathrm{~m}^{3} / \mathrm{h}$. The PAT head at each operating point was evaluated using Equation (1). For numerical simulations, the mesh quality of computational domains is important, as it affects the totally discrete Navier-Stokes (N-S) equations and measurement accuracy. Figure 4 and Table 2 display four different grid numbers used to perform the grid-sensitivity analysis. It can be established that the head showed a slight decrease after increasing the grids beyond (Grid C) $2.9 \times 10^{6}$.

$$
H=\frac{\Delta P}{\rho g}
$$






Figure 4. Grid-sensitivity analysis.

Table 2. Grid-sensitivity analysis.

\begin{tabular}{ccc}
\hline Grid & Grid Number $\left(\times \mathbf{1 0}^{\mathbf{6}}\right)$ & Head $(\mathbf{m})$ \\
\hline A & 2.2 & 8.64 \\
B & 2.57 & 9.31 \\
C & 2.9 & 9.6 \\
D & 3.41 & 9.52 \\
\hline
\end{tabular}

\subsection{Grid Convergence Index (GCI)}

Table 3 displays four different grid numbers employed for the research into PAT. In every numerical analysis, the establishment of grid convergence is essential. Thus, following the steps outlined by Celik et al. [29] to estimate and report the discretization error in CFD applications, three grids have been created (coarse, medium, fine). For the entire numerical analysis, Grid C (fine grid) was chosen.

Table 3. Four different scenarios for analyzing the Grid Convergence Index (GCI).

\begin{tabular}{cccc}
\hline Scenario & Grids $\left(\times \mathbf{1 0}^{\mathbf{6}}\right)$ & Head & GCI (\%) \\
\hline A & 2.2 & 8.64 & 8.5475 \\
B & 2.57 & 9.31 & 8.4569 \\
C & 2.9 & 9.6 & 2.1567 \\
D & 3.41 & 9.52 & 9.8534 \\
\hline
\end{tabular}

In Table 4, details of grid scenario $C$ are presented. The optimized case $C$ of the PAT (turbine mode) was meshed with estimated grid sizes using the same blocking method.

Table 4. Details for the structured grid scenario C.

\begin{tabular}{ccccc}
\hline \multirow{2}{*}{ Locations } & Grids & \multicolumn{3}{c}{ Criterions } \\
\cline { 2 - 5 } & $\mathbf{( \times \mathbf { 1 0 } ^ { \mathbf { 6 } } )}$ & Determinant $\mathbf{3} \times \mathbf{3} \times \mathbf{3}$ & Angle $\left.\mathbf{(}^{\circ}\right)$ & Aspect Ratio \\
\hline Inlet & 0.1 & $0.71-1$ & $45.88-90.64$ & $1.25-42.1$ \\
Impeller & 1.1 & $0.62-1$ & $78.24-140.6$ & $1.22-32.4$ \\
Volute & 1.2 & $0.58-1$ & $68.42-119.88$ & $1.28-42.8$ \\
Back cavity & 0.3 & $0.72-1$ & $52.77-96.44$ & $1.42-45.3$ \\
Front cavity & 0.2 & $0.65-1$ & $49.24-89.84$ & $1.20-35.4$ \\
\hline
\end{tabular}




\subsection{Numerical Simulation}

Taking into account the three-dimensional flow in the PAT mode, the numerical simulation was performed by ANSYS CFX. The shear stress transport (SST) k- $\omega$ turbulence model was selected with a $5 \%$ turbulent intensity in the steady simulation of the turbine mode. The working fluid considered for the numerical calculations was water for the turbine mode. The standard temperature of the water used was $25^{\circ} \mathrm{C}$. The static pressure of 1 atm and mass flow rate of $25 \mathrm{~m}^{3} / \mathrm{h}$ were chosen to be the inlet and output boundary conditions, respectively. The wall roughness of every boundary of each domain was defined as a "smooth wall", while the mass and momentum setting was set to "no-slip wall". The impeller was set to operate at $1450 \mathrm{r} / \mathrm{min}$.

\section{Optimization Process}

The optimization process incorporated the approach of Latin hypercube sampling (LHS), ANN, GRNN, Neuro-Fuzzy, PBGA, and numerical simulations, as shown in Figure 5. Firstly, the most influential design variables on the PAT efficiency were determined with respect to the design targets. Secondly, the DOE was performed to create the impeller details followed by the steady-state simulation. The surrogate model was then developed based on the hydraulic efficiencies attained under each operating condition. A Multiple Linear Regression (MLR) analysis provided the influence of the design variables on the efficiency. The best combination of parameters was obtained through the use of PBGA to solve the surrogate model. The pros of this scheme were to significantly save time in finding the best combination with minimal use of computational resources.

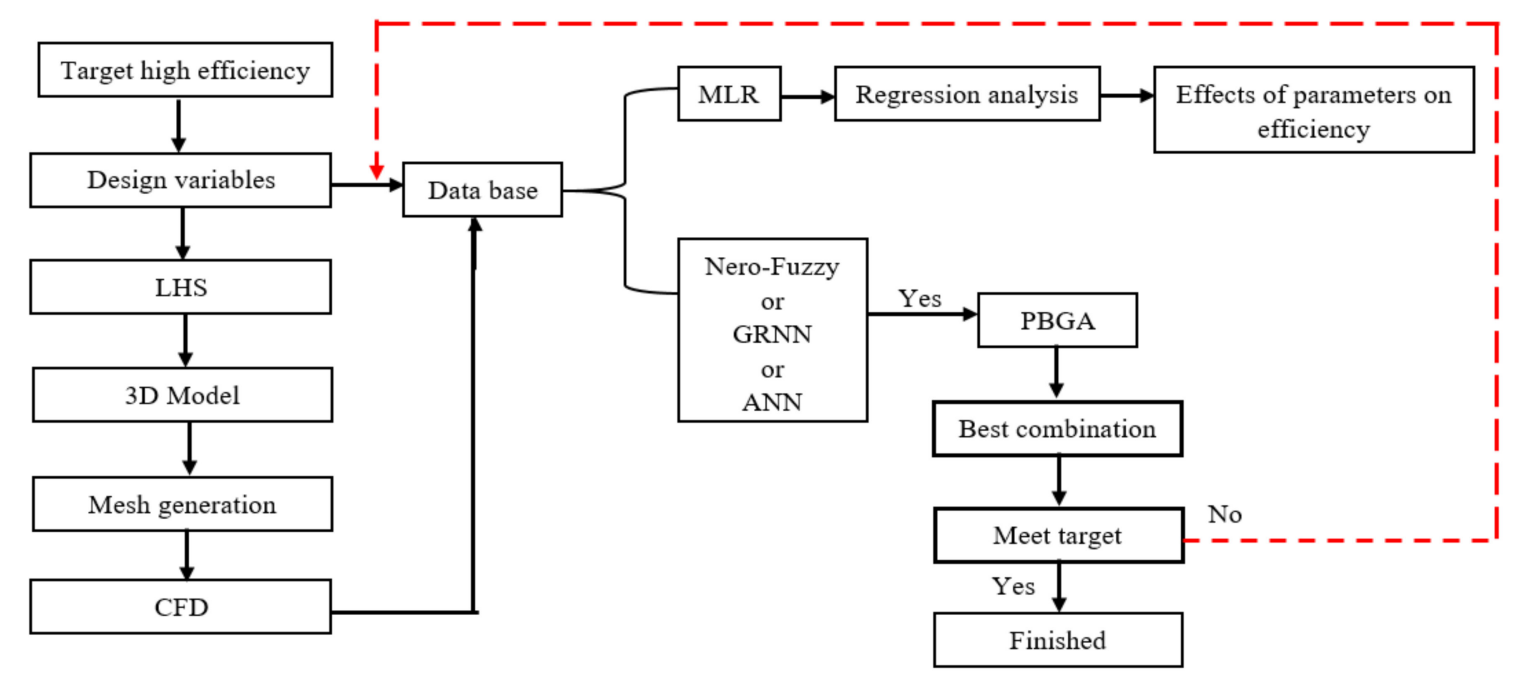

Figure 5. Optimization flow chart.

\subsection{Optimization Objective}

It aimed to maximize the turbine mode efficiencies $(\eta t)$ at a part load of $0.6 Q_{d}$, design point of $1.0 Q_{d}$ and overload of $1.4 Q_{d}$ simultaneously.

$$
\eta=\max \left(\frac{P_{0.6}}{\rho g Q_{0.6} H_{0.6}}, \frac{P_{1.0}}{\rho g Q_{1.0} H_{1.0}}, \frac{P_{1,4}}{\rho g Q_{1.4} H_{1.4}}\right),
$$

where $\rho$ and $g$ denote the water density and gravity, respectively. $Q, H$, and $P$ denote flow rate, head, and mechanical power, respectively, and they are calculated by the numerical simulation. Subscripts $0.6,1.0$, and 1.4 , respectively, represent the part load $0.6 Q_{d}$, design point $1.0 Q_{d}$ and the overload condition $1.4 Q_{d}$. 


\subsection{Design Variables}

Because the design space and impeller geometric parameters have a great impact on the efficiency of PAT [30], the blade outlet width $b_{2}$, blade exit angles $\beta_{1}$ and $\beta_{2}$, and blade number $z$ were chosen as the input design variables, as shown in Table 5.

Table 5. Ranges of design parameters.

\begin{tabular}{cccc}
\hline Design Variables & Lower & Original & Upper \\
\hline$b_{2} / \mathrm{mm}$ & 0.010 & 0.012 & 0.015 \\
$\beta_{2} /^{\circ}$ & 20 & 30 & 35 \\
$\beta_{1} /^{\circ}$ & 20 & 30 & 35 \\
$z$ & 4 & 6 & 8 \\
\hline
\end{tabular}

\subsection{Latin Hypercube Sampling Method}

The Latin hypercube sampling (LHS) method [31] was employed to design the optimization space. As introduced by Mckay et al. [31] for the LHS technique detail, it provides a randomized plan with projections on each axis for design variables that are uniformly spread. 50 cases of the impeller were randomly generated with the LHS. Some of the designed combinations of variables are listed in Table 6, which shows the objective function values in the optimization process calculated by ANSYS-CFX.

Table 6. Designed schemes and objective values.

\begin{tabular}{|c|c|c|c|c|c|c|c|}
\hline $\begin{array}{l}\text { Design } \\
\text { Scheme }\end{array}$ & $b_{2}$ & $\beta_{2}$ & $\beta_{1}$ & $\mathbf{z}$ & $\begin{array}{c}\text { PAT } \\
\eta\left(0.6 Q_{d}\right)\end{array}$ & $\begin{array}{c}\text { PAT } \\
\eta\left(1.0 Q_{d}\right)\end{array}$ & $\begin{array}{c}\text { PAT } \\
\eta\left(1.4 Q_{d}\right)\end{array}$ \\
\hline 1 & 0.014 & 28.787 & 31.154 & 5 & 49.98 & 74.23 & 83.41 \\
\hline 2 & 0.014 & 28.350 & 22.219 & 5 & 81.06 & 76.50 & 91.62 \\
\hline 3 & 0.012 & 25.726 & 26.575 & 5 & 61.41 & 83.24 & 74.24 \\
\hline 4 & 0.011 & 20.369 & 20.446 & 7 & 62.38 & 82.88 & 63.93 \\
\hline 5 & 0.014 & 30.399 & 24.641 & 6 & 77.53 & 54.76 & 89.79 \\
\hline 6 & 0.011 & 26.033 & 33.038 & 6 & 25.33 & 80.38 & 63.92 \\
\hline 7 & 0.010 & 27.247 & 33.987 & 8 & 14.12 & 83.81 & 70.19 \\
\hline 8 & 0.014 & 21.740 & 26.062 & 4 & 48.45 & 92.16 & 83.98 \\
\hline 9 & 0.011 & 32.670 & 21.396 & 6 & 78.82 & 65.34 & 65.80 \\
\hline$\ldots$ & $\ldots$ & $\ldots$ & $\ldots$ & $\ldots$ & $\ldots$ & $\ldots$ & $\ldots$ \\
\hline 50 & 0.010 & 29.969 & 30.427 & 5 & 46.67 & 70.11 & 68.36 \\
\hline
\end{tabular}

\subsection{Surrogate Models}

A surrogate model is an engineering approach used when an intended outcome of interest cannot be easily and directly predicted, so a representation of the result is used instead [32]. Many engineering design problems involve tests or simulations as a function of design variables to determine the design objective and constraint functions. The literature contains many surrogate models, but the most commonly used ones are the Back Propagation Artificial Neural Network (BPANN), Generalized Regression Neural Network (GRNN), kriging, Adaptive Neuro-Fuzzy Inference System (ANFIS), and Artificial Neural Network (ANN).

It can take several hours or days to run a single simulation for real-world problems. As a result, routine activities, such as system optimization, space exploration design, and sensitivity analysis, become difficult, as thousands and millions of simulation tests are required. One way to lessen this problem is to build predictive models, known as surrogate models, to mimic the simulation model's behavior as closely as possible, with the advantage of being cheaper to assess computationally.

- Artificial Neural Network Training

The concept of an Artificial Neural Network (ANN) was inspired by the biological neural networks of animal brains. It is a calculating system with an interconnected group of nodes, usually consisting 
of an input layer, one or more hidden layers, and an output layer [33]. It adopts activation functions in the neurons of the hidden layers and output layers to build a robust nonlinear relationship between the inputs and outputs [34]. The mathematical relation for the ANN function is written as Equation (3); the activation function, tanh, is written as Equation (4); and the linear function as Equation (5).

$$
\begin{gathered}
y=g\left(\sum_{j=1}^{n} w_{j}^{2} \times f\left(\sum_{k=1}^{m} w_{k^{\prime} j}^{1} x_{k}+b_{n}^{1}\right)\right)+b^{2}, \\
f(x)=\left[\frac{2}{\left(1+e^{-2 x}\right)}\right]-1, \\
g(x)=a x+b
\end{gathered}
$$

where $w$ denotes the weight coefficient, $b$ denotes the threshold, $\mathrm{m}$ and $\mathrm{n}$ denote the number of incoming layers, $j$ and $k$ denote a count from 1 to $m$ and $n$, and parameter $a$ specifies the gradient of the line (that is, how steep the line is). The superscript 1 and 2 denotes the first and second layer coefficients from the hidden to the output layer, respectively. In this study, the architecture of ANN consists of three layers, namely, input layer, hidden layer, and output layer, as indicated in Figure 6.



Figure 6. Architecture of Artificial Neural Network (ANN).

- General Regression Neural Network (GRNN)

The Generalized Neural Regression Network (GRNN), proposed by D.F. Specht [17], is a single-pass neural network in the hidden layer that uses a Gaussian activation function. GRNN is composed of input, hidden, summation, and division layers. Using Equation (5), we can obtain the regression of the random variable $\mathrm{y}$ on the observed values $\mathrm{X}$ of random variable $\mathrm{x}$.

$$
E|y| x \mid=\frac{\int_{-\infty}^{\infty} y f(x, y) d y}{\int_{-\infty}^{\infty} f(x, y) d y}
$$

where $f(x, y)$ is a known function of the joint continuous probability density. If $f(x, y)$ is unknown, a set of observations of $x$ and $y$ should be calculated. The non-parametric consistent estimator suggested by Parzen in Equation (6) may be used to estimate $f(x, y)$.

$$
\hat{f}(x, y)=\frac{1}{2 \pi(p+1) / 2 \sigma(p+1)} \frac{1}{n} \sum_{i=1}^{n} e^{-\frac{\left(x-x^{i}\right)^{T}\left(x-x^{i}\right)}{2 \sigma^{2}}} e^{-\frac{\left(y-y^{i}\right)^{2}}{2 \sigma^{2}}},
$$

where $y^{i}$ is the desired scalar output given the observed input of $x^{i}, n$ is the number of training pairs $\left(x^{i} \rightarrow\right.$ $\left.y^{i}\right), p$ is the dimension of the vector variable, $T$ denotes the transpose, and $\sigma$ is the smoothing parameter.

In this study, the GRNN architecture is comprised of four layers, as indicated in Figure 7. 


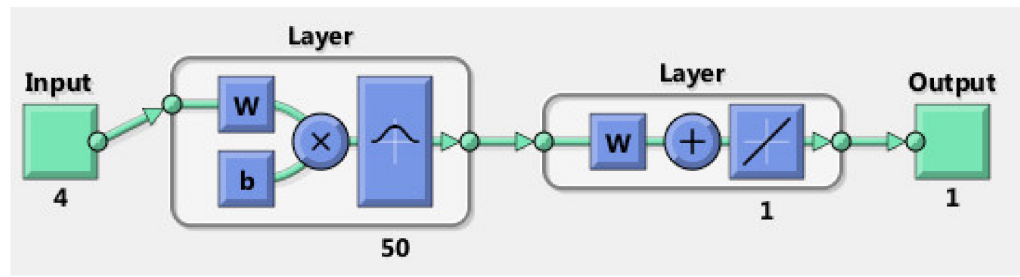

Figure 7. Architecture of Generalized Regression Neural Network (GRNN).

- $\quad$ Adaptive Neuro-Fuzzy Inference System (ANFIS)

The shortcomings of the Fuzzy Logic System [16] and Neural Networks [35] are to be solved by the Adaptive Neuro-Fuzzy Inference System (ANFIS), which is the blend of the Fuzzy Logic System and the neural network technology. ANFIS is a simple technique of data learning using a Fuzzy Logic model to turn a particular input into a target outcome through highly connected processing elements of the network and communication information, weighted to map the numerical inputs onto an output [36]. The most important advantage of using ANFIS is that all its parameters can be trained in a Fuzzy Logic system as a Neural Network [37]. The hybridization of ANN and FIS is done by applying a learning algorithm for mapping input-outputs to confirm the optimization of the parameters used in the development of the FIS. Figure 8 [38] shows the architecture of a standard ANFIS system, which has two input variables with five layers. The Matlab neuro-fuzzy software offers a simple platform for ANFIS predictions.

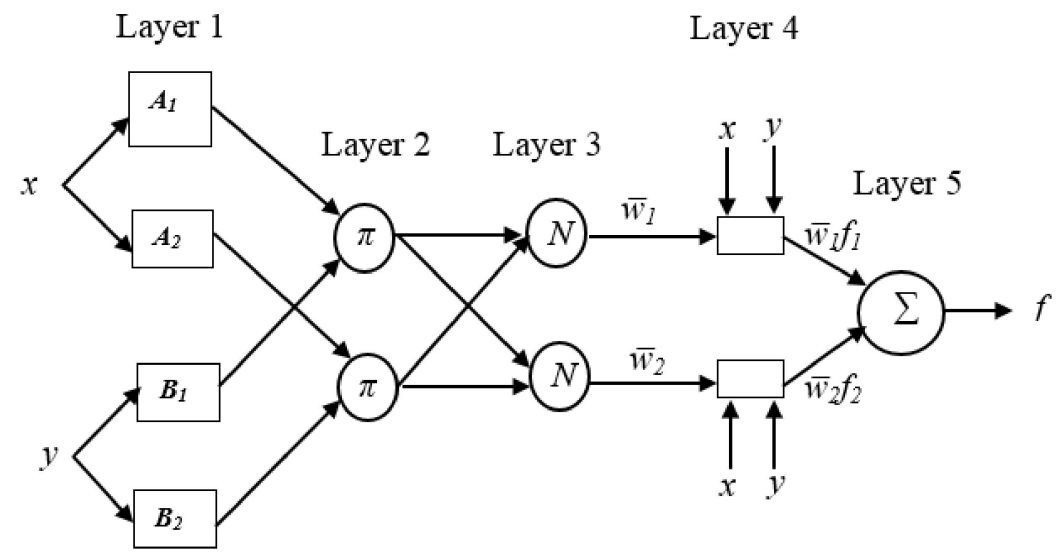

Figure 8. Adaptive Neuro-Fuzzy Inference System (ANFIS) architecture.

The application herein is for prediction of the PAT efficiency at a flow rate of $0.6 Q_{d}, 1.0 Q_{d}$, and $1.4 Q_{d}$. The epoch was set to 100 so that the number of iterations was adequate during the learning process. Figure 9 shows the Adaptive Neuro-Fuzzy Inference System (ANFIS) predicting the design variables' performance. As shown in Figure 9, blade width, blade exit angles, and blade number $\left(b_{2}, \beta_{2}\right.$, $\beta_{1}$ and $z$ ) are the inputs to the fuzzy-inference system, while the obtained efficiencies derived from defuzzification is the output. The model structure for PAT efficiency, as illustrated in Figure 10, consists of four inputs, three membership functions for each input, and one output.

For solving the influence of the design variables over the objective functions, the Sugeno fuzzy-inference system was used. The CFD efficiencies obtained from PAT at $0.6 Q_{d}, 1.0 Q_{d}$, and $1.4 Q_{d}$ operating conditions were used as training data to train the ANFIS network with Gaussian membership function through a hybrid learning algorithm. The information used in ANFIS for this study is listed in Table 7. By applying the ANFIS tool which incorporates the fuzzy IF-THEN rules, the total rules generated by the input data of the established models for the membership functions were 81 ; the first ten of the rules have been depicted in the Appendix A. Such conditional statements explain how the results were constructed in conjunction with the three membership functions implemented. 




Figure 9. View of the developed fuzzy model.

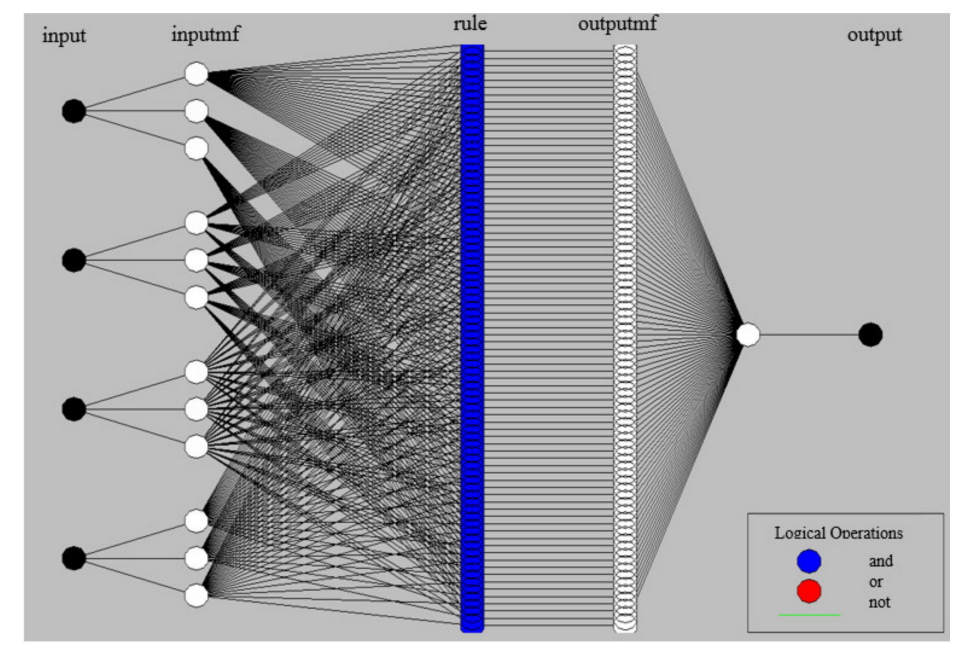

Figure 10. ANFIS model structure.

Table 7. ANFIS information used for solving the influence of the design variables over the objective functions.

\begin{tabular}{cccc}
\hline Variable & \multicolumn{3}{c}{ PAT Efficiency } \\
\cline { 2 - 4 } & $\mathbf{0 . 6} \boldsymbol{Q}_{\boldsymbol{d}}$ & $\mathbf{1 . 0} \boldsymbol{Q}_{\boldsymbol{d}}$ & $\mathbf{1 . 4 Q _ { \boldsymbol { d } }}$ \\
\hline Number of nodes & 193 & 193 & 193 \\
Number of linear parameters & 405 & 405 & 405 \\
Number of nonlinear parameters & 24 & 24 & 24 \\
Total number of parameters & 429 & 429 & 429 \\
Number of training data pairs & 50 & 50 & 50 \\
Number of checking data pairs & 0 & 0 & 0 \\
Number of fuzzy rules & 81 & 81 & 81 \\
\hline
\end{tabular}

The ANFIS model regarding input and output variables is shown in a three-dimensional (3-D) mesh plot in Figure 11. The surface plots display both the connecting lines and axes of the surface in color. The (3-D) surface plots obtained from ANFIS explains the relation between the output and two inputs. Figure 11a-f shows some of the surface plots for ANFIS networks relating inputs to PAT efficiency. It can be concluded that the ANFIS model generates a smooth surface, proving a high precision of the model.

It can also be concluded from the surface plot that the influence of the design variables toward obtaining the output can be easily obtained through the ANFIS algorithm. Some algorithms demand massive computational resources to obtain a similar result. All the surface plots show that the total surface is rule-base covered.

It can be also deduced from Figure 11 that the optimization problem is non-convex. A non-convex optimization involves an objective function that has multiple optima, only one of which is the global optima. Depending on the surface plot, it can be very difficult to locate the global optima. Due to that, the Pareto-based genetic algorithm was employed in this study to solve the multi-objective 
optimization. Genetic algorithms [39] are optimization techniques developed in 1975 by J. Holland, who was inspired by Charles Darwin's theory of the biological population's evolution. The genetic algorithms are based on the principle of the survival of the fittest by using genetic operators such as selection, crossover, and mutation. The fitness of a particular individual is measured using a fitness function, which evaluates how close the individual is to the objective [40,41]. In a multi-objective problem, the objectives can be conflicting. A single solution is hardly the best for all the objectives simultaneously. Instead of a single optimum, there is a set of trade-off solutions, generally known as Pareto-optimal solutions [42]. These solutions are optimal in the sense that no other solutions in the design space are better than them or can "dominate" them when all the objectives are considered.



(a)

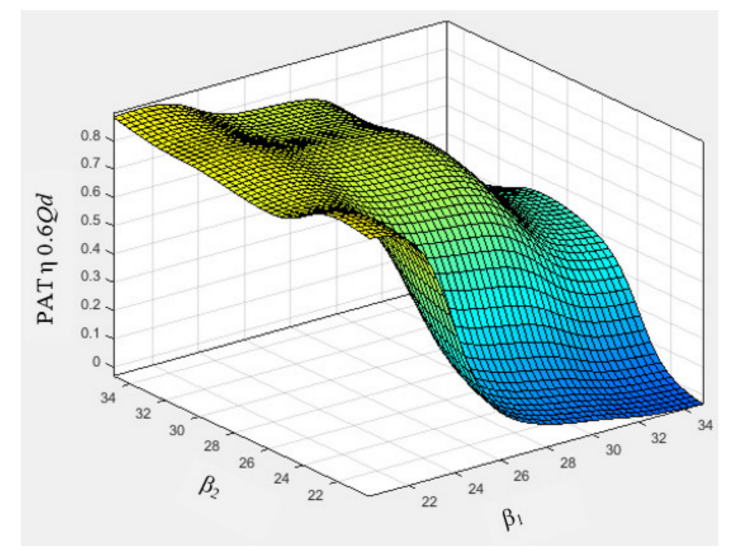

(b)

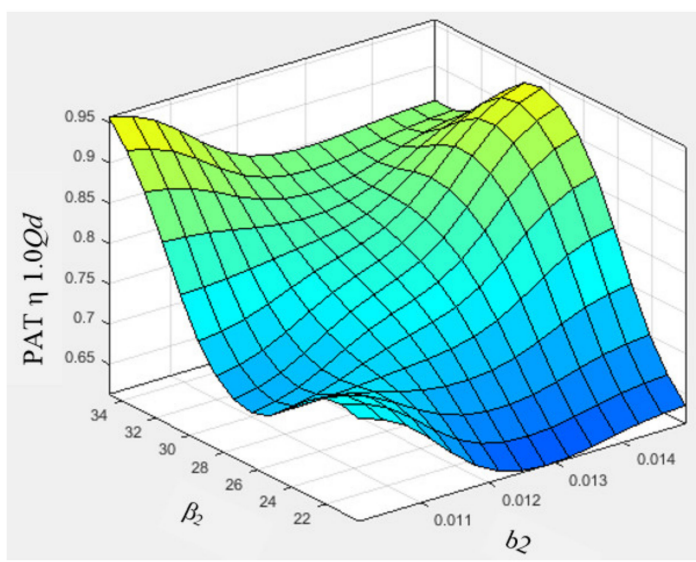

(c)

Figure 11. Cont. 


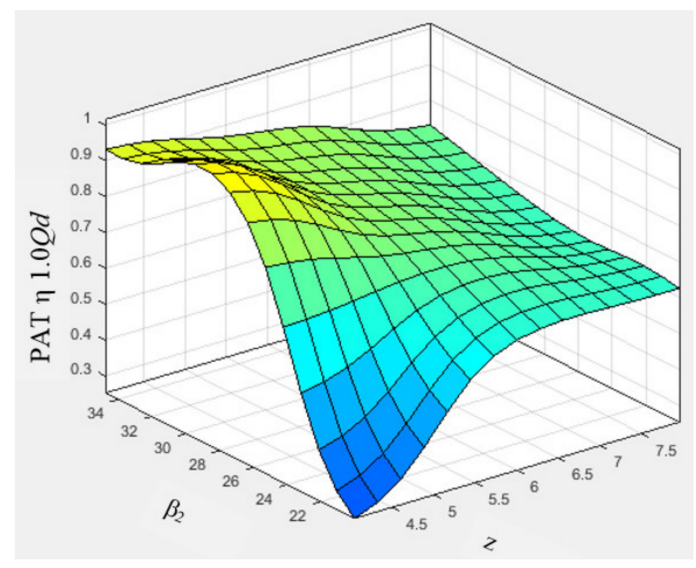

(d)



(e)

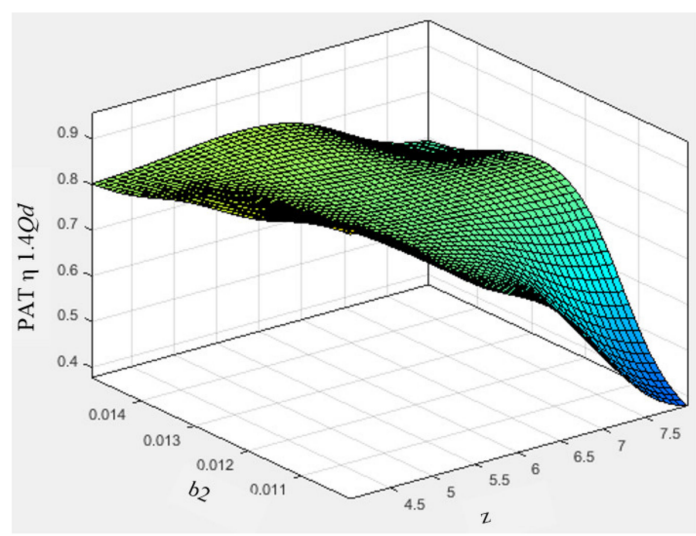

(f)

Figure 11. (a-f) Surface view of Sugeno-type ANFIS.

\subsection{Multi-Objective Optimization}

When there are two or more objective functions within the same variable constraints, then a multi-objective optimization is used to maximize or minimize the objective functions. A Pareto-optimal optimization was performed because obtaining a solution that would optimize all objectives is practically impossible. This is because, usually, at least one parameter becomes worse off when any other parameter is improved. For these conditions, the Pareto frontier, a solution set for applying all Pareto efficiency, was used to decide on the optimal parameter combinations. The Pareto-based genetic algorithm (PBGA) was applied to reach the frontier [43]. The Matlab function code gamultiobj [44] was used; this function can be used to solve multi-objective optimization problems with several objective 
functions, making it advantageous over the simple genetic algorithm, and it has been used effectively in optimization research [45-47]. The fitness function for each individual was then derived from the surrogate model to update the Pareto frontier of each iteration, including the initial population. The optimization problem is stated by:

$$
\text { find }\left\{\begin{array}{c}
\text { maximize } \eta_{t}\left(0.6 Q_{d}\right)=f_{1}\left(b_{2}, \beta_{2}, \beta_{1}, z\right) \\
\eta_{t}\left(1.0 Q_{d}\right)=f_{1}\left(b_{2}, \beta_{2}, \beta_{1}, z\right) \\
\eta_{t}\left(1.4 Q_{d}\right)=f_{1}\left(b_{2}, \beta_{2}, \beta_{1}, z\right) \\
\text { s.t } \\
0.010 \leq b_{2} \geq 0.015 \\
20 \leq \beta_{2} \geq 35 \\
20 \leq \beta_{1} \geq 35 \\
4 \leq z \geq 8
\end{array},\right.
$$

Since the objective functions were known to be more than one, the value of each function could not be used to evaluate the individuals. The expression in Equation (9) was applied [48] as:

$$
F(x)=\left[\frac{1}{1+\|x-y\|_{2}}\right],
$$

where $x$ denotes any single individual, $y$ denotes the Pareto individual closet to $x$, and $\|x-y\|$ denotes the Euclidean distance. The following input parameters have been used in the development of the Pareto-optimal solutions: a population size of 100 , a Pareto-front population of 0.8 , a crossover fraction of $0.85,1000$ generations, and a function tolerance of 10 .

\section{Results and Discussion}

\subsection{Statistical Analysis}

A regression analysis was used to build predictive equations to determine the cause-effect relationship between the variables in a given set of data. Multiple Linear Regression (MLR) is an approach used to explain how a dependent variable is described by several independent variables [49]. MLR can be mathematically formulated as:

$$
y_{i}=\beta_{0}+\beta_{1} x_{i 1}+\beta_{2} x_{i 2}+\cdots+\beta_{p} x_{i p},
$$

where, for $i=n$ observations: $y_{i}=$ dependent variable, $x_{i}=$ explanatory variables, $\beta_{0}=y$-intercept (constant term), $\beta_{p}=$ slope coefficients for each explanatory variable, and $\epsilon=$ the model's error term. The degree of linearity is computed using decision coefficient, whereas the error term represents the difference between the estimated variables and the real ones [50].

An analysis of variance showing the influence of the design variables $\left(b_{2}, \beta_{2}, \beta_{1}\right.$, and $\left.z\right)$ on the objective functions $\left(0.6 Q_{d}, 1.0 Q_{d}\right.$, and $\left.1.4 Q_{d}\right)$ in this regression analysis are presented in Tables 8-10. In the tables, DF denotes the degree of freedom, which is the number of independent variables in the regression model. SS denotes the sum of the square of the difference between the predicted value and mean of the value of all the data points. MS denotes the mean of the sum of squares or the sum of squares divided by the degrees of freedom for both regression and residuals. The F-value is obtained by dividing the Mean Square Regression by the Mean Square Residual. The P-value is the probability of obtaining results as extreme as the observed results of a statistical hypothesis test, assuming that the null hypothesis is correct. Evidently, the variables have a large impact in evaluating the objective functions. The test statistics of the PAT efficiency for $0.6 Q_{d}, 1.0 Q_{d}$, and $1.4 Q_{d}$ have F-values of 116.61 , 
101.56 , and 152.57 respectively, which is larger than the critical value $F_{0.05,4,45}=2.82$. This analysis shows that the means of the variables vary greatly in statistical terms.

Table 8. Variance for PAT $\eta$ at $0.6 Q_{d}$.

\begin{tabular}{cccccc}
\hline Source & DF & SS & MS & F-Value & $p$-Value \\
\hline Regression & 4 & 2.32229 & 0.58057 & 116.61 & 0.000 \\
\hline Error & 45 & 0.22404 & 0.00498 & - & - \\
\hline Total & 49 & 2.54633 & - & - & - \\
\hline
\end{tabular}

Table 9. Variance for PAT $\eta$ at $1.0 Q_{d}$.

\begin{tabular}{cccccc}
\hline Source & DF & SS & MS & F-Value & $p$-Value \\
\hline Regression & 4 & 0.61078 & 0.152696 & 101.56 & 0.000 \\
\hline Error & 45 & 0.06766 & 0.001503 & - & - \\
\hline Total & 49 & 0.67844 & - & - & - \\
\hline
\end{tabular}

Table 10. Variance for PAT $\eta$ at $1.4 Q_{d}$.

\begin{tabular}{cccccc}
\hline Source & DF & SS & MS & F-Value & $p$-Value \\
\hline Regression & 4 & 0.63893 & 0.159733 & 152.57 & 0.000 \\
\hline Error & 45 & 0.04711 & 0.001047 & - & - \\
\hline Total & 49 & 0.68605 & - & - & - \\
\hline
\end{tabular}

To establish the regression equation using multiple regression analysis, PAT geometrical parameters, i.e., blade exit width, blade exit angles, and blade number $\left(b_{2}, \beta_{2}, \beta_{1}\right.$, and $\left.z\right)$, were selected as the independent variables. The PAT efficiencies at part load, design point, and overload $\left(0.6 Q_{d}, 1.0 Q_{d}\right.$, and $\left.1.4 Q_{d}\right)$ were also selected as the dependent variables.

In Table 11, the Multiple Linear Regression model summarized for PAT efficiencies at $0.6 Q_{d}, 1.0 Q_{d}$, and $1.4 Q_{d}$ is shown. It can be observed that the adjusted $\mathrm{R}$-squared value for $1.4 Q_{d}$ is higher than for $0.6 Q_{d}$ and $1.0 Q_{d}$. The R-squared value of $90.42,89.14$, and 92.52 implies that $90.42 \%, 89.14 \%$, and $92.52 \%$ of the data fit the regression model at part load $\left(0.6 Q_{d}\right)$, design point $\left(1.0 Q_{d}\right)$, and overload $\left(1.4 Q_{d}\right)$ flow conditions, respectively. The standard errors of the input variables were very minimal for all the operating conditions except exit blade width $\left(b_{2}\right)$.

Table 11. Summary of regression analysis.

\begin{tabular}{ccccccc}
\hline & $\mathbf{0 . 6} Q_{d}$ & \multicolumn{2}{c}{$\mathbf{1 . 0} Q_{d}$} & \multicolumn{2}{c}{$\mathbf{1 . 4 Q _ { d }}$} \\
\hline Variable & Value & Std. Error & Value & Std. Error & Value & Std. Error \\
\hline Constant & 0.403 & 0.123 & 0.6606 & 0.0721 & 0.7919 & 0.0604 \\
\hline$b_{2}$ & 33.72 & 7.39 & 35.90 & 3.87 & 40.11 & 3.32 \\
\hline$\beta_{2}$ & 0.02669 & 0.00240 & 0.01126 & 0.00129 & 0.00674 & 0.00110 \\
\hline$\beta_{1}$ & -0.03841 & 0.00243 & -0.01417 & 0.00129 & -0.01105 & 0.00110 \\
\hline$z$ & 0.00540 & 0.00882 & -0.04634 & 0.00484 & -0.07027 & 0.00416 \\
\hline & Adjusted $\mathrm{R}^{2} 90.42$ & & Adjusted $\mathrm{R}^{2}$ & 89.14 & Adjusted $\mathrm{R}^{2}$ 92.52 \\
\hline
\end{tabular}

Regression equations of PAT $\eta$ at the part load, design point, and overload have been expressed in Equation (11), (12), and (13), respectively.

$$
0.6 Q_{d}=0.403+33.72 b_{2}+0.02669 \beta_{2}-0.03841 \beta_{1}+0.00540 z,
$$




$$
\begin{aligned}
& 1.0 Q_{d}=0.6606+35.90 b_{2}+0.01126 \beta_{2}-0.01417 \beta_{1}-0.04634 z \\
& 1.4 Q_{d}=0.7919+40.11 b_{2}+0.00674 \beta_{2}-0.01105 \beta_{1}-0.07027 z
\end{aligned}
$$

With a higher $\mathrm{R}^{2}$ value between the input and the output and a minimal standard deviation error, it can be concluded that the input variables have a significant influence on the output variables.

\subsection{Prediction Results From ANN, GRNN, and ANFIS}

- $\quad$ Artificial Neural Network (ANN) Prediction Results

The regression diagrams of the CFD and the ANN values are shown in Figure 12. The fit line signifies the CFD data, which indicates a great adjustment between the ANN model and the CFD. The correlation coefficient R-square is used to evaluate the accuracy of the model. The R-square values for the PAT efficiencies at $0.6 Q_{d}, 1.0 Q_{d}$, and $1.4 Q_{d}$ were calculated as $0.9966,0.99837$, and 0.999 , respectively. As indicated in Figure $13 \mathrm{a}-\mathrm{c}$ which denotes the PAT efficiencies at $0.6 Q_{d}, 1.0 Q_{d}$, and $1.4 Q_{d}$ respectively, it is concluded that the ANN model is able to predict the results fairly well compared to the CFD simulation.

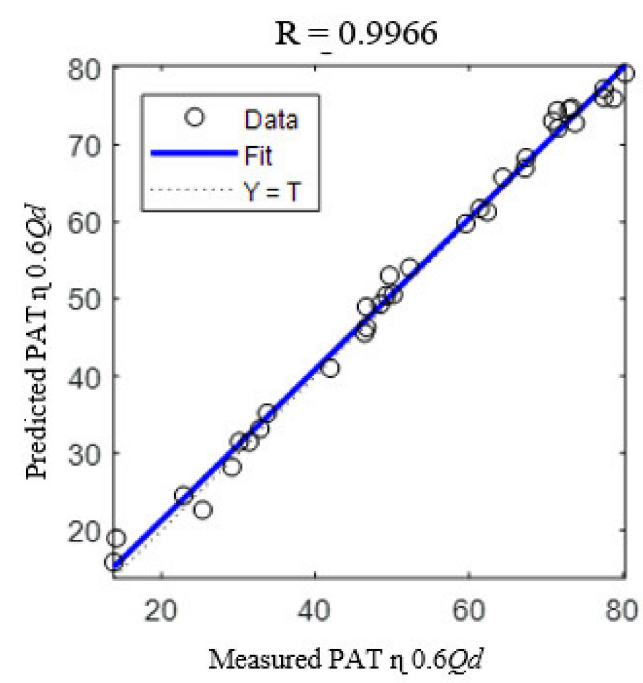

(a)

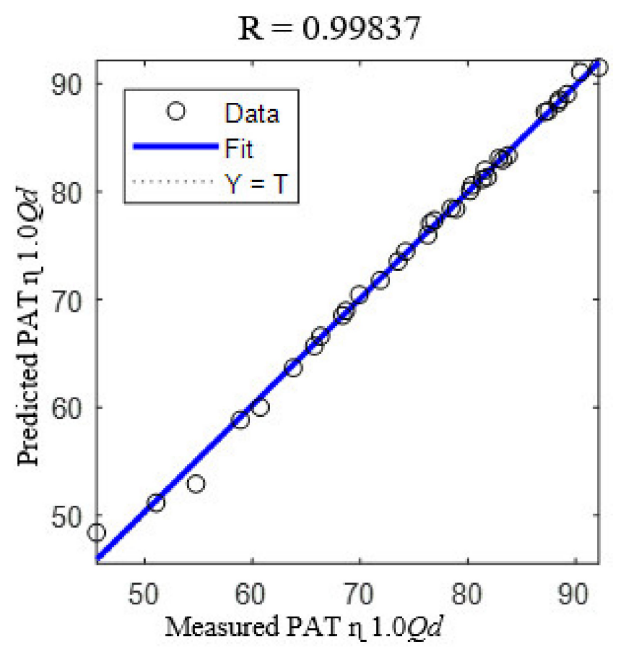

(b)

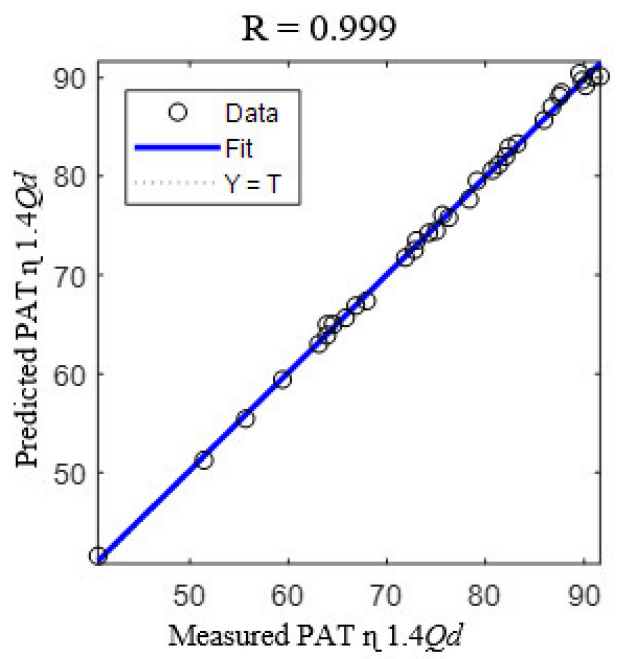

(c)

Figure 12. R-square analysis of PAT efficiency: (a) $0.6 Q_{d}$, (b) $1.0 Q_{d}$, and (c) $1.4 Q_{d}$. 


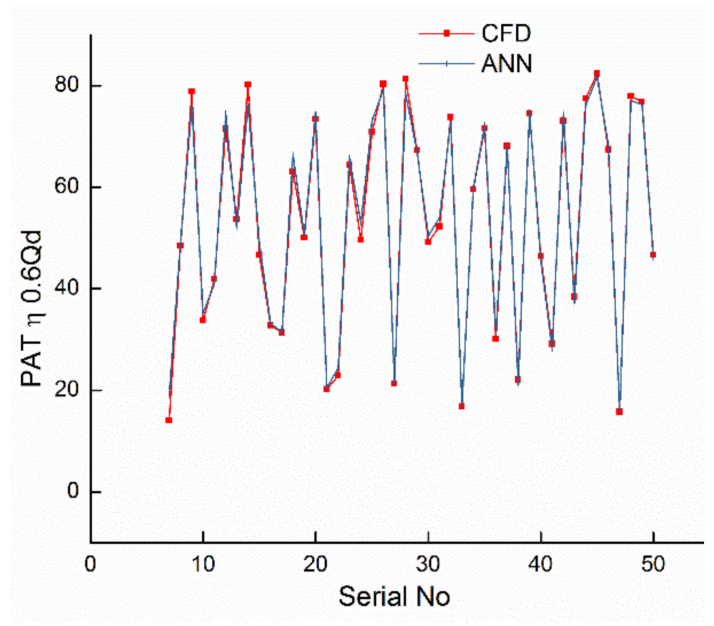

(a)

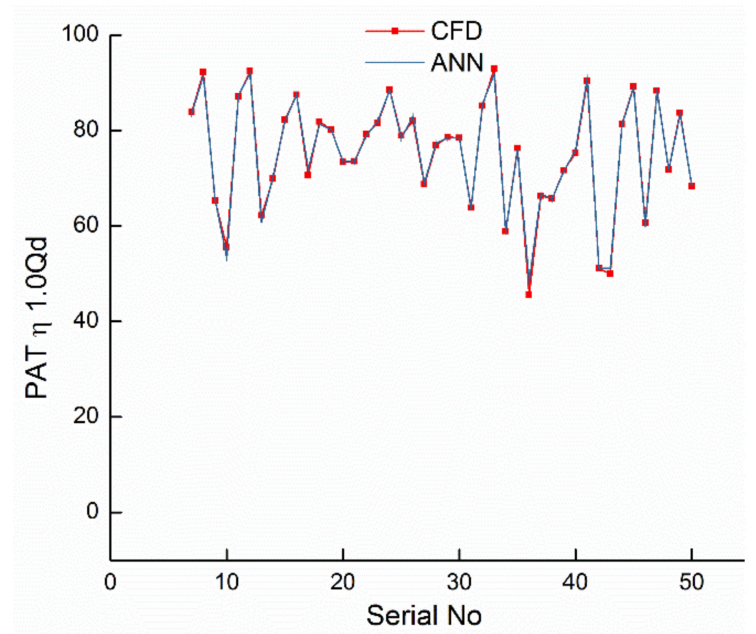

(b)



(c)

Figure 13. Validation of ANN prediction with CFD: (a) $0.6 Q_{d},(\mathbf{b}) 1.0 Q_{d}$, and (c) $1.4 Q_{d}$

\section{- $\quad$ GRNN Network Prediction Results}

The regression diagrams of the CFD and GRNN-predicted values are displayed in Figure 14. The fit line denotes the CFD data, which specify a large amendment between the GRNN model and the CFD results. The R-square values for PAT efficiencies at $0.6 Q_{d}, 1.0 Q_{d}$, and $1.4 Q_{d}$ were calculated as $0.98396,0.97695$, and 0.86996 , respectively. From Figure $15 a-c$ representing PAT efficiencies at $0.6 Q_{d}$, $1.0 Q_{d}$, and $1.4 Q_{d}$ respectively, it can be established from the outcomes the good agreement between the GRNN model and the CFD simulation.

- $\quad$ Adaptive Neuro-Fuzzy Inference System (ANFIS)

Figure 16 displays the R-square of objective functions for the ANFIS model. The R-square values for PAT efficiencies at $0.6 Q_{d}, 1.0 Q_{d}$, and $1.4 Q_{d}$ were calculated as $0.9999,1$, and 0.99998 , respectively. As indicated in Figure $17 \mathrm{a}-\mathrm{c}$ depicting PAT efficiencies at $0.6 Q_{d}, 1.0 Q_{d}$, and $1.4 Q_{d}$ respectively, it can be concluded that the predicted ANFIS model agrees well with the CFD values. Correlations between the observed and predicted data for $0.6 Q_{d}, 1.0 Q_{d}$, and $1.4 Q_{d}$ demonstrated the capability of the ANFIS model prediction. 




(a)

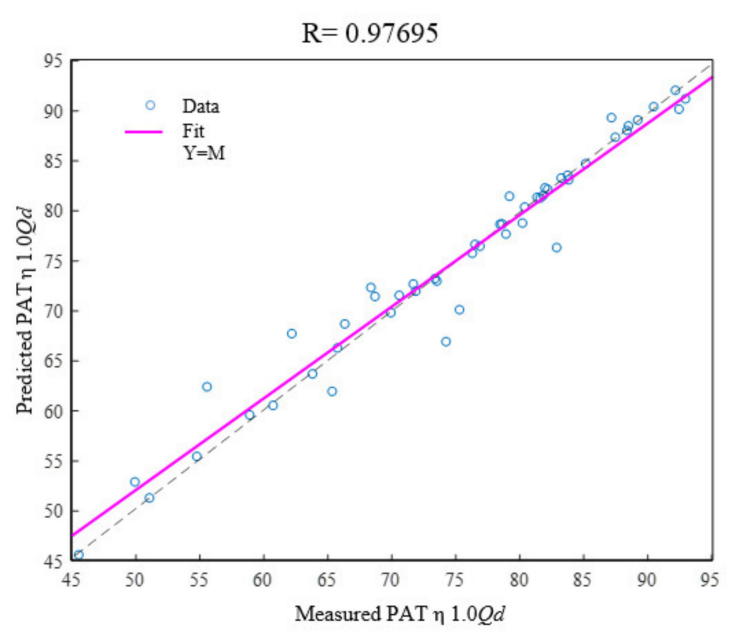

(b)

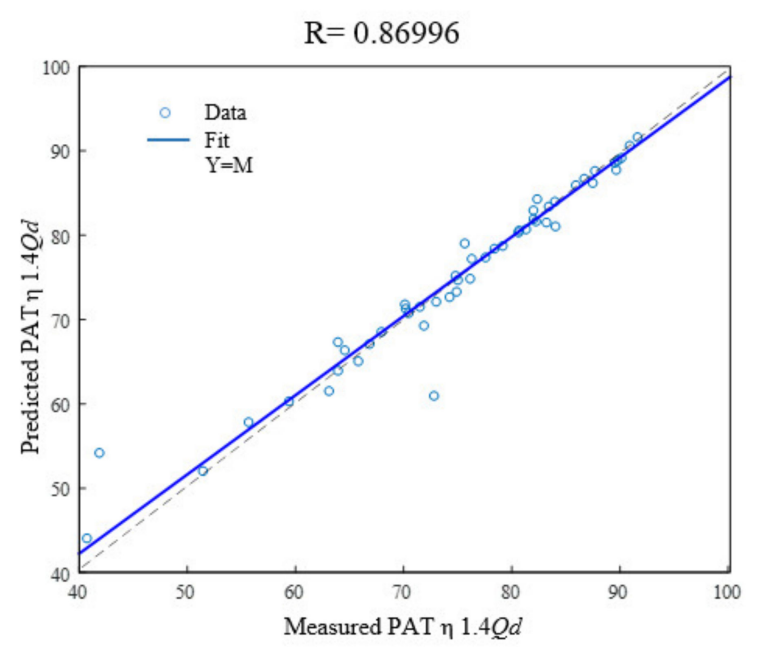

(c)

Figure 14. R-Square analysis of PAT efficiency: (a) $0.6 Q_{d}$, (b) $1.0 Q_{d}$, and (c) $1.4 Q_{d}$.

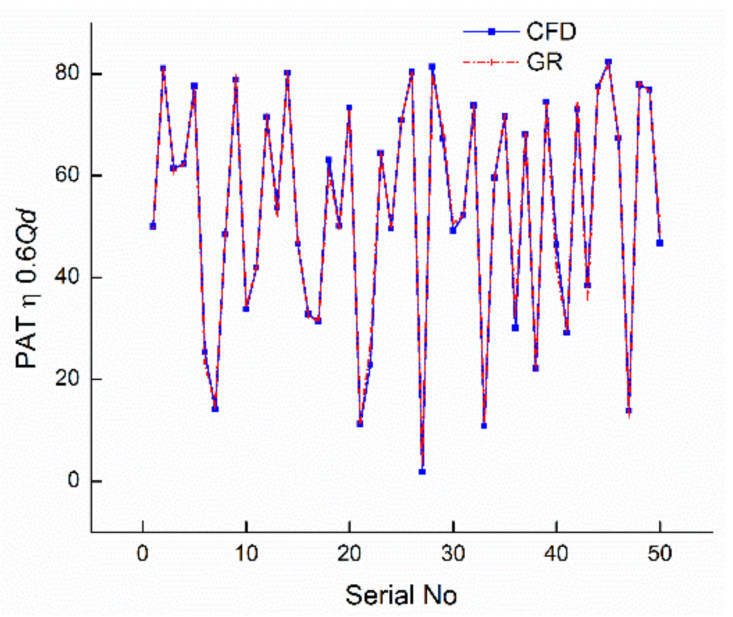

(a)

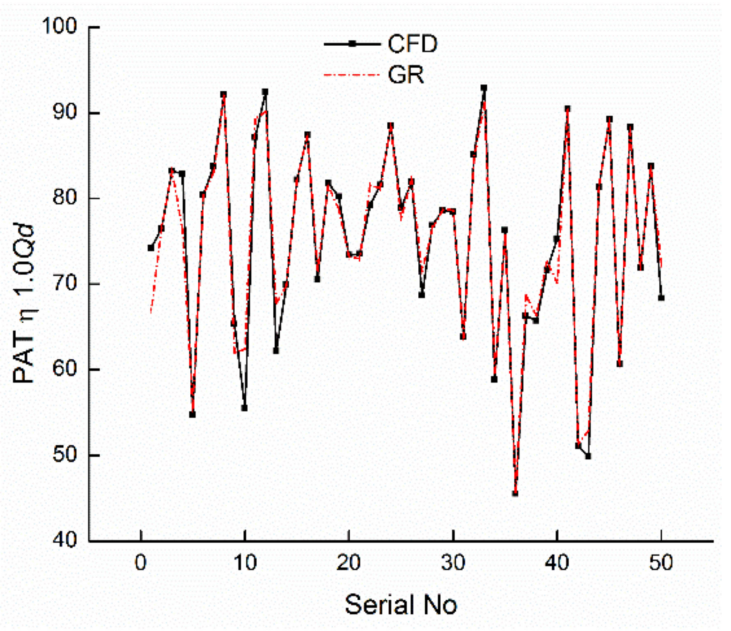

(b)

Figure 15. Cont. 


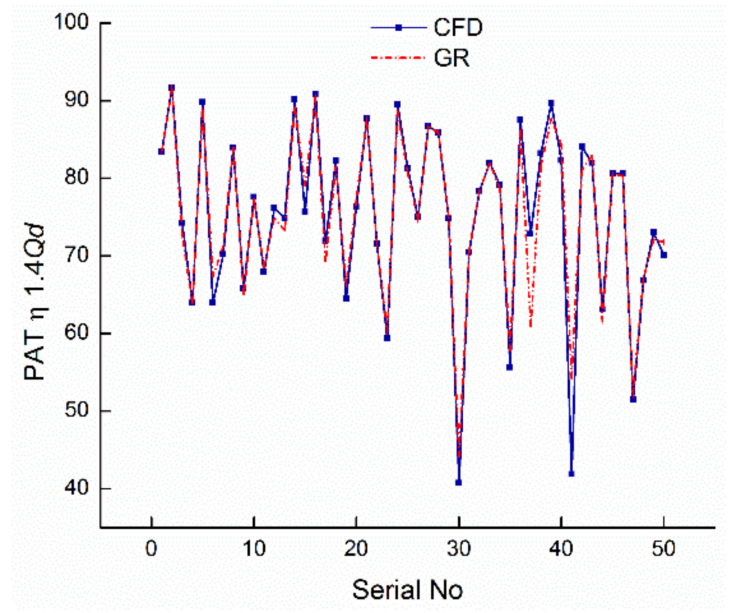

(c)

Figure 15. Validation of GRNN prediction with CFD: (a) $0.6 Q_{d}$, (b) $1.0 Q_{d}$, and (c) $1.4 Q_{d}$.

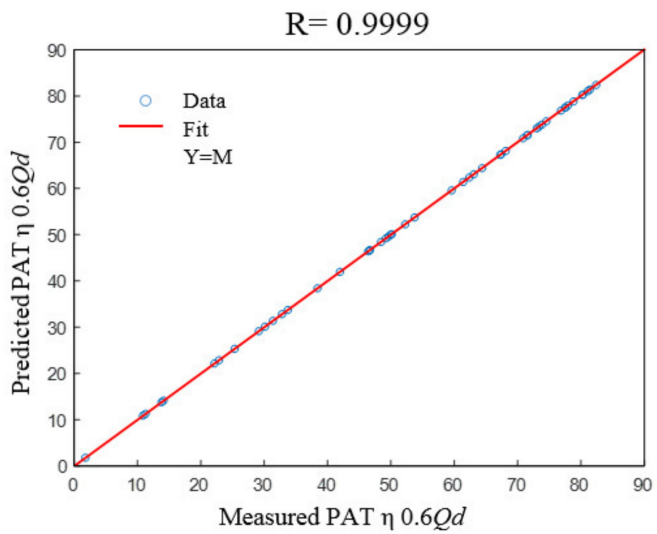

(a)

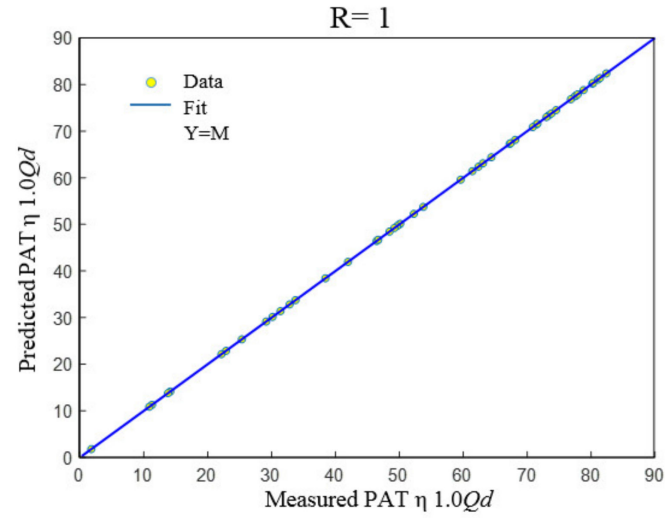

(b)

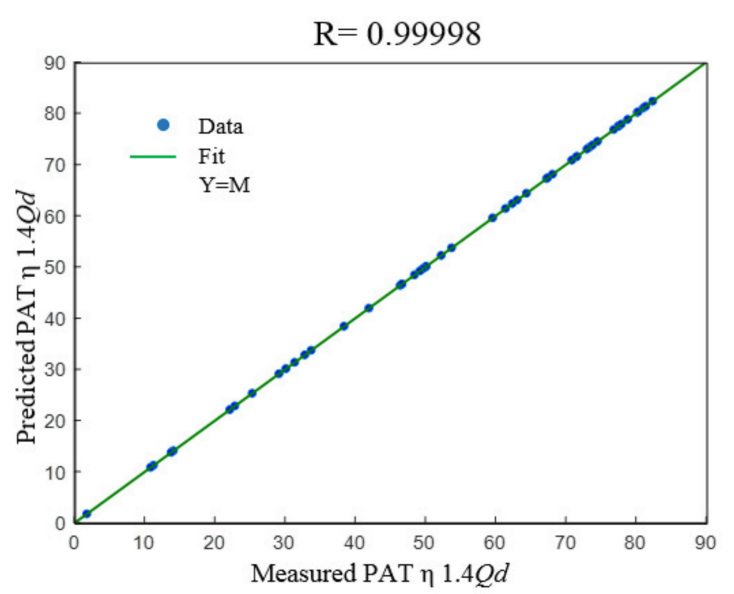

(c)

Figure 16. R-Square analysis of PAT efficiency: (a) $0.6 Q_{d}$, (b) $1.0 Q_{d}$, and (c) $1.4 Q_{d}$. 




(a)

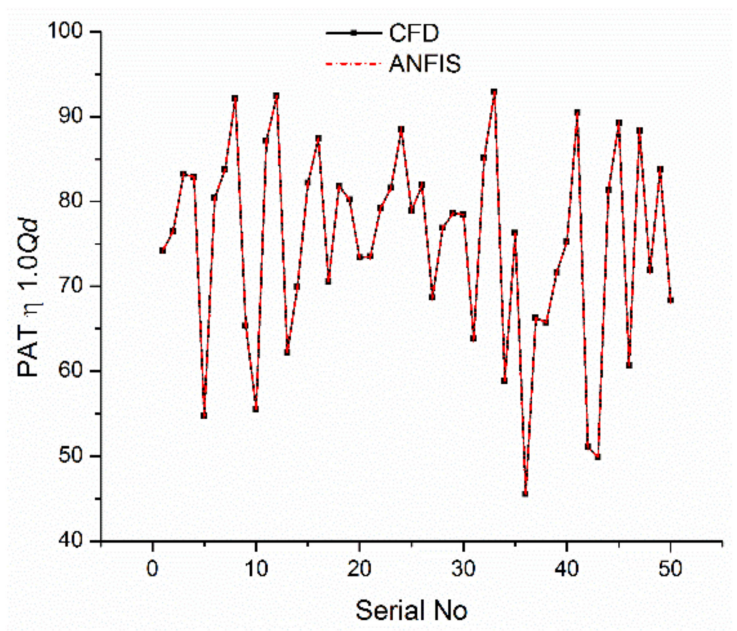

(b)

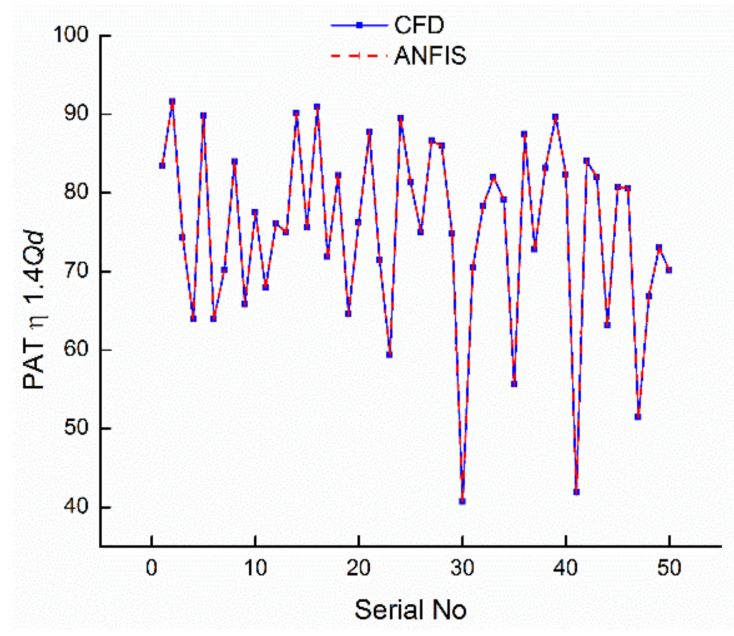

(c)

Figure 17. Validation of ANFIS prediction with CFD: (a) $0.6 Q_{d}$, (b) $1.0 Q_{d}$, and (c) $1.4 Q_{d}$.

- Comparison Between the Surrogate Models

The average training and test errors in the present analysis were correlated with the findings obtained from the ANFIS, ANN, and GRNN predictions of the objective functions. The root mean square of error (RMSE) was chosen to evaluate the accuracy of the prediction models. Table 12 shows the RMSE obtained from both models.

Table 12. Root mean square of error (RMSE) comparison for ANFIS, ANN, and GRNN.

\begin{tabular}{ccccccc}
\hline \multirow{2}{*}{ Model } & \multicolumn{2}{c}{$\mathbf{0 . 6} Q_{d}$} & \multicolumn{2}{c}{$\mathbf{1 . 0 Q _ { d }}$} & \multicolumn{2}{c}{$\mathbf{1 . 4 Q _ { d }}$} \\
\cline { 2 - 7 } & Training & Testing & Training & Testing & Training & Testing \\
\hline ANFIS & 0.00053 & 0.0065 & 0.000358 & 0.00414 & 0.000473 & 0.000544 \\
ANN & 0.0068 & 0.0074 & 0.0063 & 0.00863 & 0.00681 & 0.00771 \\
GRNN & 0.124 & 0.680 & 0.547 & 0.980 & 0.723 & 1.048 \\
\hline
\end{tabular}

From the table, the ANFIS models achieved an extremely small average error which, however, was slightly large for the ANN and GRNN models. The comparative result confirmed the superiority of ANFIS over ANN and GRNN. Nevertheless, the computational cost and complexity of the ANFIS prediction model were extremely high when compared to the ANN and GRNN prediction models. 
However, by comparing the ANN and GRNN prediction models, the ANN had a higher prediction accuracy than the GRNN. Owing to that, ANN was adopted for the multi-objective optimization of the PAT impeller.

\subsection{Solutions to the Three-Objective Problem}

The Matlab function gamultiobj [44] was employed to solve the Pareto solutions. The Pareto frontier for the three objective optimizations is shown in Figure 18. The blue points are the Pareto optimum front, which is the optimum solution set of optimization. Each point represents a model impeller. The Pareto solutions presented a set of 1000 optimized generated impellers that satisfied the optimization objectives. Three best impellers were selected with the optimum decision variables, as shown in Table 13.

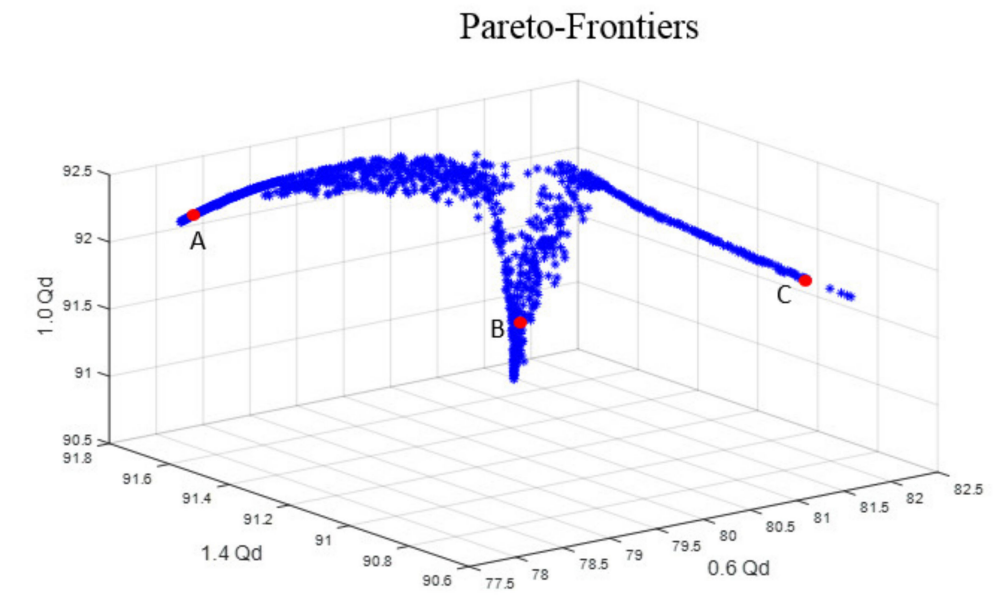

Figure 18. Pareto-frontiers from GRNN.

Table 13. Variables for optimum cases.

\begin{tabular}{cccccccc}
\hline Case & $\boldsymbol{b}_{\mathbf{2}}$ & $\boldsymbol{\beta 2}$ & $\boldsymbol{\beta 1}$ & $\mathbf{z}$ & $\begin{array}{c}\text { PAT } \\
\eta\left(\mathbf{0 . 6} \boldsymbol{Q}_{d}\right)\end{array}$ & $\begin{array}{c}\text { PAT } \\
\eta\left(\mathbf{1 . 0} Q_{d}\right)\end{array}$ & $\begin{array}{c}\text { PAT } \\
\eta\left(\mathbf{1 . 4} Q_{d}\right)\end{array}$ \\
\hline $\mathrm{A}$ & 0.015 & 24 & 20.14 & 4 & 77.7 & 92.3 & 91.6 \\
\hline $\mathrm{B}$ & 0.014 & 29.75 & 20.20 & 4 & 80.7 & 92.2 & 91.4 \\
\hline $\mathrm{C}$ & 0.015 & 30.75 & 20.06 & 5 & 82.1 & 91.8 & 90.9 \\
\hline
\end{tabular}

Table 14 presents the CFD results from the optimized cases. The CFD predictions were very close to the 3-D Pareto predictions at the design and overload conditions, rendering the results valid. The effect of the optimization was slightly less for all the optimized cases at part load.

Table 14. Comparison of baseline model and the three optimized models.

\begin{tabular}{|c|c|c|c|c|c|c|c|}
\hline Case & $b_{2}$ & $\beta 2$ & $\beta 1$ & $\mathbf{z}$ & $\begin{array}{c}\text { PAT } \\
\eta\left(0.6 Q_{d}\right)\end{array}$ & $\begin{array}{c}\text { PAT } \\
\eta\left(1.0 Q_{d}\right)\end{array}$ & $\begin{array}{c}\text { PAT } \\
\eta\left(1.4 Q_{d}\right)\end{array}$ \\
\hline model & 0.012 & 30 & 30 & 6 & 50.01 & 73.92 & 78.59 \\
\hline A & 0.015 & 24 & 20.14 & 4 & 54.07 & 81.37 & 85.93 \\
\hline B & 0.014 & 29.75 & 20.20 & 4 & 56.52 & 83.58 & 86.94 \\
\hline C & 0.015 & 30.75 & 20.06 & 5 & 61.88 & 82.41 & 86.74 \\
\hline
\end{tabular}

Figure 19 presents a comparison of the baseline model and the three optimized cases at PAT mode. At part load, optimized case $C$ performed better than the other cases. Optimized case $C$ yielded an efficiency increase of $23.7 \%$, while the optimized cases A and B increase by $8.1 \%$ and $13 \%$, respectively, 
in the inefficiency. At overload condition, optimized case B performed slightly better, with a $10.6 \%$ increase in efficiency, higher than optimized cases A and C that produced a $9.34 \%$ and $10.4 \%$ increase, respectively. At the design point, optimized case B performed better, with a $13.1 \%$ increase in efficiency as compared to cases $\mathrm{A}$ and $\mathrm{C}$, with a $10.1 \%$ and $11.5 \%$ respective increase in inefficiency.

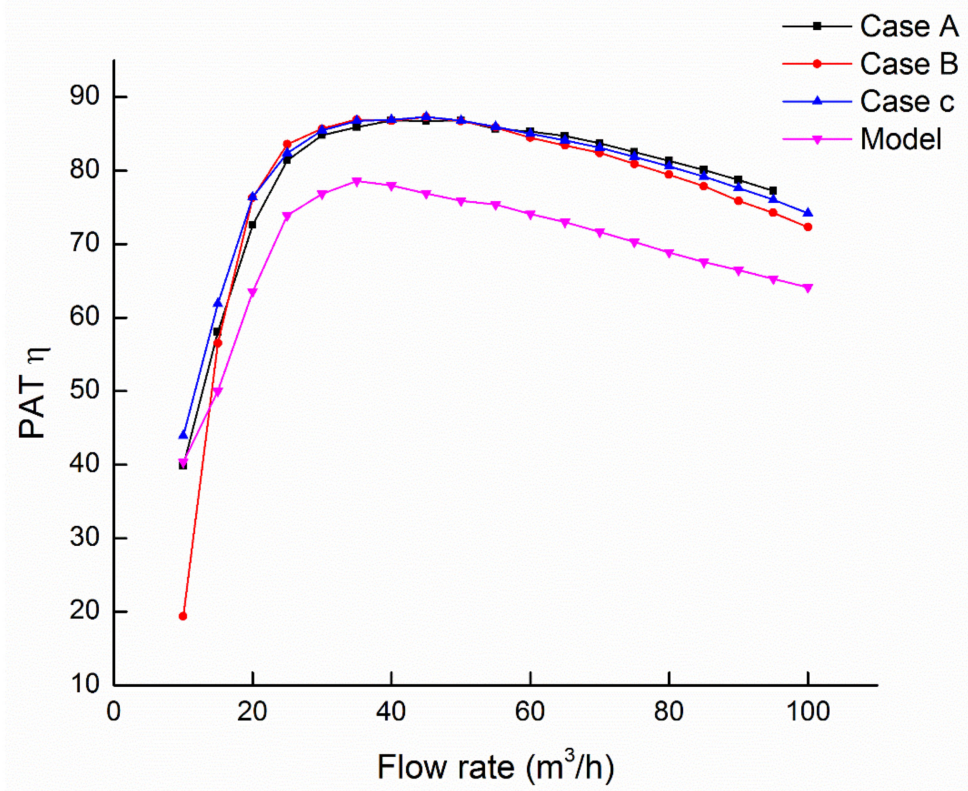

Figure 19. Predicted PAT efficiency comparison at different flow rates.

Comparing the three cases in Figure 19, it is glaringly obvious that the best single case for the optimization objective is case C. PAT is characterized for its poor performance at part load, and among all the three optimized cases, optimized case $C$ offers an improved efficiency at part load, design point, and under overload condition. Based on this, it can also be deduced from the original case that increasing $b_{2}$ from $0.012 \mathrm{~mm}$ to $0.015 \mathrm{~mm}, \beta_{2}$ from $30^{\circ}$ to $30.75^{\circ}$, reducing $\beta_{1}$ from $30^{\circ}$ to $20.06^{\circ}$, and reducing the blade number from six to five can enhance the hydraulic performance at the part load, design, and overload flow conditions, as depicted in Figure 20.

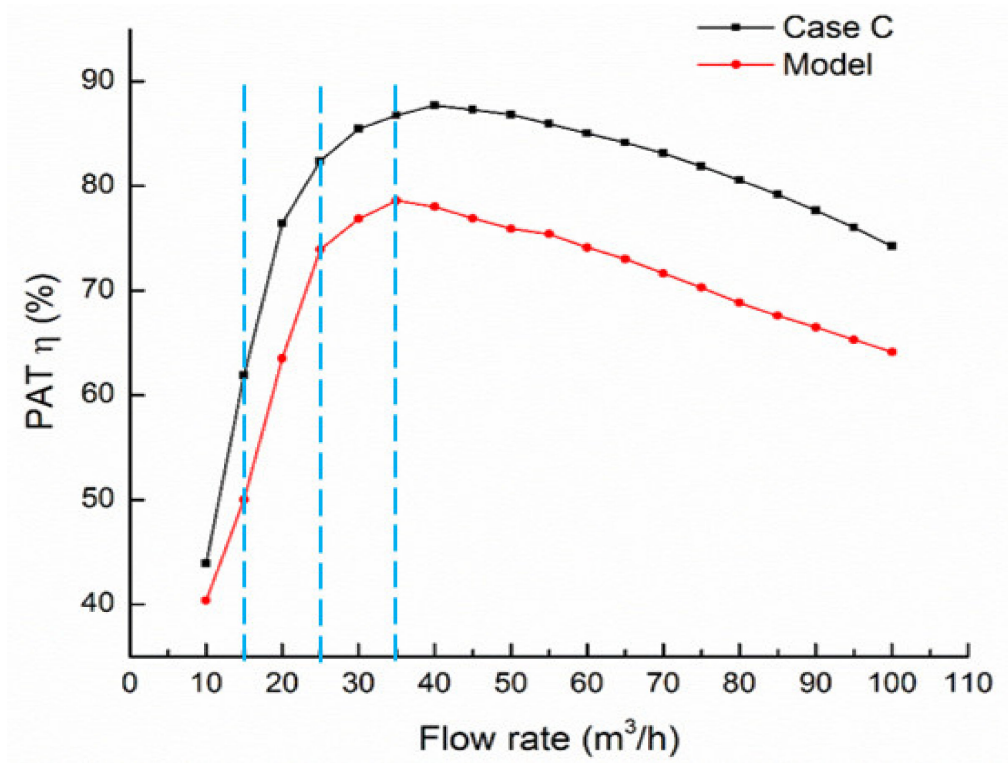

Figure 20. Optimal PAT case C PAT efficiency comparison at different flow rates. 


\subsection{Internal Flow Analysis}

The internal flow of the optimized impeller case (C) was compared with the original model to make known the influence of optimization on the flow structure. Figure 21 gives a comparison of the pressure distribution on the blade surface for the three optimized flow conditions. At a $0.6 Q_{d}$ flow rate, the static pressure for the original and optimized blades was almost uniform. At the outlet of the impeller, the static pressure was the lowest, while the inlet of the impeller recorded the highest pressure. The differences in static pressure in the flow passage were relatively small. This could probably explain why the optimization effect was lesser at part load.

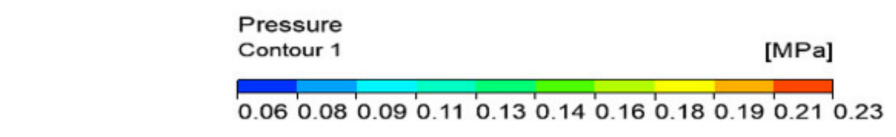



$0.6 Q_{d}$

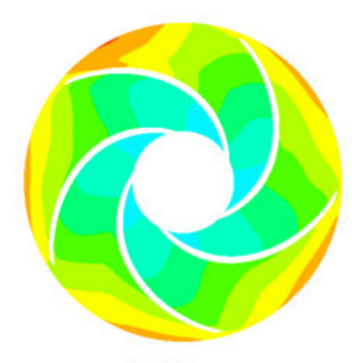

$0.6 Q_{d}$

Original Impeller

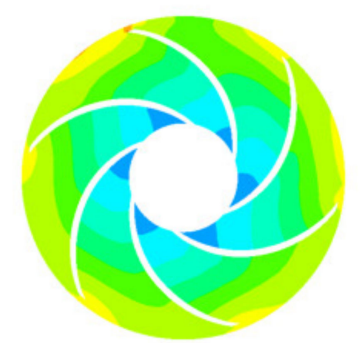

$1.0 Q_{d}$
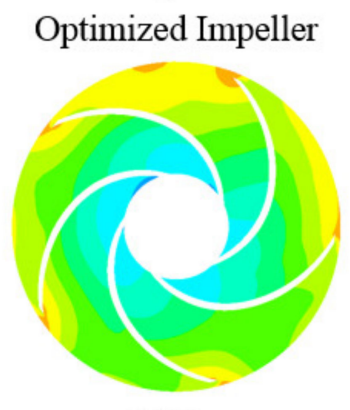

$1.0 Q_{d}$



$1.4 Q_{d}$

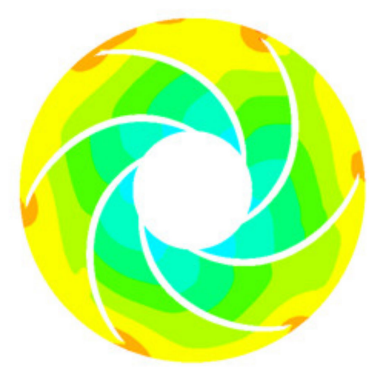

$1.4 Q_{d}$

Figure 21. Comparative pressure distribution results for the baseline and optimized impellers.

For the design condition, the original model had a low static pressure region at the outlet, which indicated a likelihood of the occurrence of cavitation. This phenomenon could reduce the hydraulic performance of the PAT. After optimization, the low-pressure areas at the blade leading regions were reduced, indicating an improvement in the cavitation performance. At overload condition, the static pressure was high at the impeller inlet regions, which could probably be attributed to the increase in flow rate. After optimization, the static pressure was uniformly distributed. For the three flow conditions, the low pressure distribution on the blade leading regions and the high pressure at the trailing regions reduced significantly after the optimization; this could improve the hydraulic performance.

The streamline distribution in the impeller flow channel was also analyzed for the various flow conditions, as shown in Figure 22. At part load condition, the streamlines of the PAT model were disordered and vortices manifested between the leading region and the mid-section of the impeller, which could cause severe energy loss and a secondary flow. However, the streamlines at the design conditions were slightly smooth. The streamlines of the impellers at overload conditions were also disordered and could cause severe energy loss and a secondary flow. For the optimized impellers, at part load, design point and overload flow conditions, the flow was uniform, and there was no vortex or separation. The streamline in the channels was uniform in all the optimized models. 


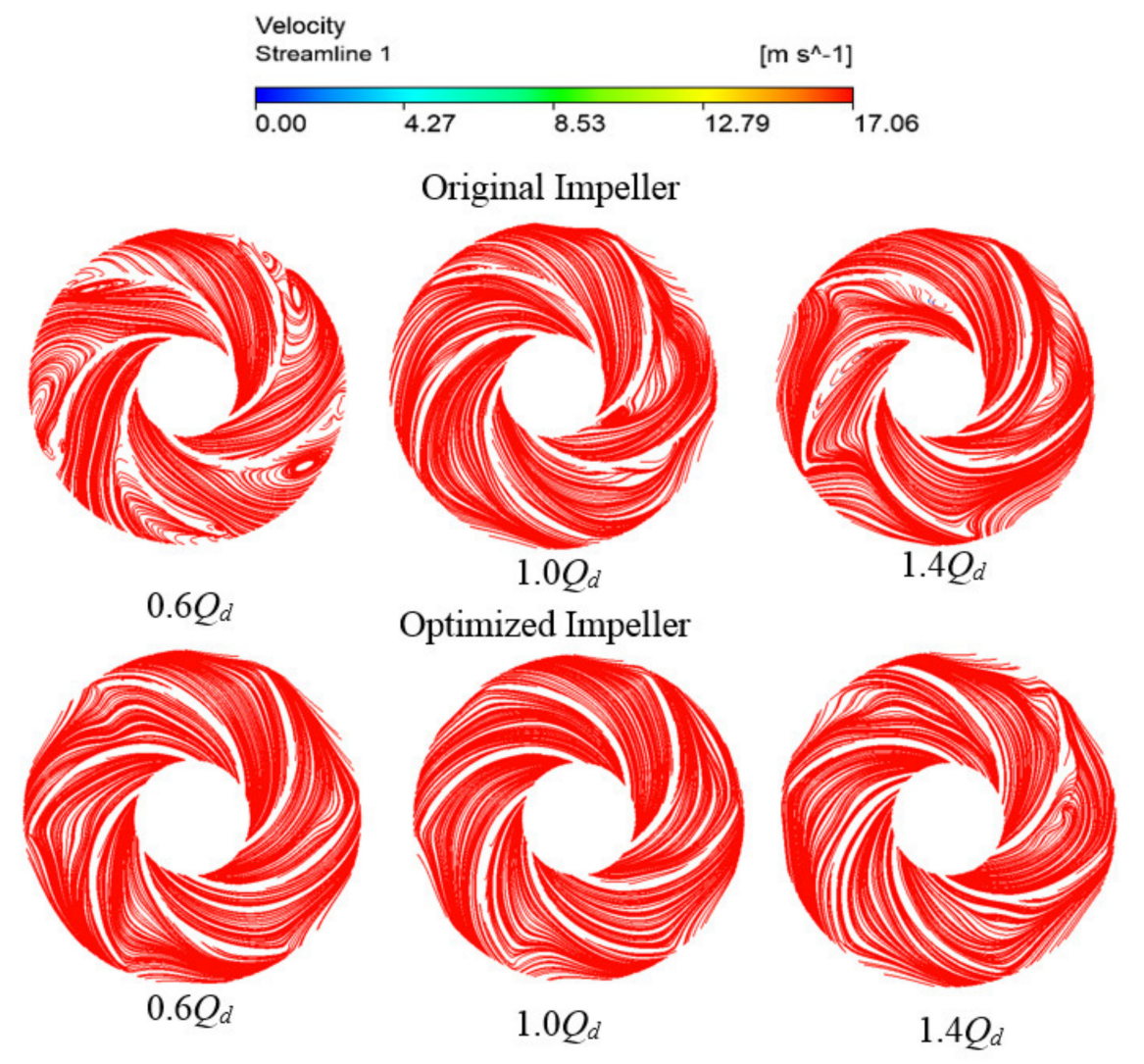

Figure 22. Compared streamlines on blade surface.

\section{Conclusions}

The PAT impeller optimization is a very important process in reducing hydraulic losses to improve turbine mode hydraulic performance. However, designing a physical model for such phenomena is often costly and requires high expertise. Therefore, machine learning techniques such as surrogate models were used to discover a hidden relationship between the objective functions and the design variables. Achieving a reliable surrogate model for predicting the PAT performance can save time, energy, and cost. In this study, Artificial Neural Network (ANN), General Regression Neural Network (GRNN), and Adaptive Neuro-Fuzzy Inference System (ANFIS) algorithms were used to develop a predictive model for the objective function of the PAT. To improve the hydraulic performance of the PAT over a broader operating range, a suitable surrogate model for the optimization was first determined, followed by a multi-conditional optimization. The conclusions are as follows:

(1) In predicting the objective functions, learning the design variables with ANN and GRNN was simpler and computationally cost-effective when compared to ANFIS.

(2) The impeller parameter prediction by ANFIS was very complex and tedious, making it computationally expensive for modeling and multi-objective optimization.

(3) The simulation findings indicated that the ANN-PBGA model proposed by this study had advantageous properties, such as better precision fitting; it is more suitable for small samples and offers a stronger nonlinear fitting capability.

(4) The PAT with the optimized impeller had a higher efficiency than that of the original PAT model. The optimized design variables of PAT were enhanced respectively by $23.7 \%, 11.5 \%$, and $10.4 \%$ at part load, design point, and overload conditions.

(5) After the numerical simulation of the optimized designed impeller under part load, design condition, and overload, the static pressure distribution and streamlines were analyzed. For the 
optimized case $C$, the static pressure was uniformly distributed and the streamlines were smooth, with a very low magnitude of vortices in all the flow conditions.

Nevertheless, there is a need for further research into the surrogate model for other applications. The PBGA-ANN model should be tested and further improved for other engineering fields. Theoretical investigation, which enables the effect of modifications to non-convex optimization, should be extensively researched. Additionally, it is not possible to find the global optimum of every non-convex problem due to (nondeterministic polynomial time) the NP-hardness barrier.

Author Contributions: Original draft, S.N.A.; funding acquisition, J.Y.; data accuracy, L.W.; review \& editing, D.A. and K.A.A.-P. All authors have read and agreed to the published version of the manuscript.

Funding: This research was funded by: China Postdoctoral Science Foundation (Grant No. 2018M632244), Natural Science Foundation of Jiangsu Province (Grant No. BK20180879), High-level Talent Research Foundation of Jiangsu University (Grant No. 18JDG012), National Key Research and Development Plan Project (2018YFB0606103), and Construction of Dominant Disciplines in Colleges and Universities and Universities in Jiangsu (PAPD).

Conflicts of Interest: The authors declare no conflicts of interest.

\section{Nomenclature}

Symbols

$\begin{array}{ll}Q & \text { flow rate }\left(\mathrm{m}^{3} / \mathrm{h}\right) \\ \eta & \text { efficiency } \\ n_{s} & \text { specific speed } \\ \omega & \text { omega } \\ n & \text { rotational speed }(\mathrm{rpm}) \\ \mathrm{z} & \text { blade number }\end{array}$

\section{Abbreviation}

$\begin{array}{ll}\text { BEP } & \text { Best Efficiency Pump } \\ \text { SST } & \text { Shear Stress Transport } \\ 3-D & \text { 3-Dimensional } \\ N-S & \text { Navier-Stokes } \\ C F D & \text { Computational Fluid Dynamics } \\ \text { PAT } & \text { Pump-As-Turbine } \\ \text { ANFIS } & \text { Adaptive Neuro-Fuzzy Inference System } \\ \text { GRNN } & \text { General Regression Neural Network } \\ \text { NSGA } & \text { Non-dominated Sorting Genetic Algorithm } \\ \text { ANN } & \text { Artificial Neural Networks } \\ \text { BPGA } & \text { Pareto-based genetic algorithm }\end{array}$

\section{Appendix A}

1. If $\left(b_{2}\right.$ is in1mf1) and ( $\beta_{2}$ is in2mf1), $\left(\beta_{1}\right.$ is in $\left.3 \mathrm{mf} 1\right),(z$ is in4mf1), then (PAT efficiency is out1mf1) (1).

2. If $\left(b_{2}\right.$ is in $\left.1 \mathrm{mf} 1\right)$ and $\left(\beta_{2}\right.$ is in $\left.2 \mathrm{mf} 1\right),\left(\beta_{1}\right.$ is in $\left.3 \mathrm{mf} 1\right),(z$ is in $4 \mathrm{mf} 2)$, then (PAT efficiency is out $\left.1 \mathrm{mf} 2\right)(1)$.

3. If $\left(b_{2}\right.$ is in $\left.1 \mathrm{mf} 1\right)$ and $\left(\beta_{2}\right.$ is in $\left.2 \mathrm{mf} 1\right),\left(\beta_{1}\right.$ is in $\left.3 \mathrm{mf} 1\right),(z$ is in $4 \mathrm{mf} 3)$, then (PAT efficiency is out $\left.1 \mathrm{mf} 3\right)(1)$.

4. If $\left(b_{2}\right.$ is in1mf1) and ( $\beta_{2}$ is in $\left.2 \mathrm{mf} 1\right),\left(\beta_{1}\right.$ is in $\left.3 \mathrm{mf} 1\right),(z$ is in $4 \mathrm{mf} 1)$, then (PAT efficiency is out1mf4) (1).

5. If $\left(b_{2}\right.$ is in $\left.1 \mathrm{mf} 1\right)$ and $\left(\beta_{2}\right.$ is in $\left.2 \mathrm{mf} 1\right),\left(\beta_{1}\right.$ is in $\left.3 \mathrm{mf} 1\right),(z$ is in $4 \mathrm{mf} 2)$, then (PAT efficiency is out1mf5) (1).

6. If $\left(b_{2}\right.$ is in $\left.1 \mathrm{mf} 1\right)$ and ( $\beta_{2}$ is in $\left.2 \mathrm{mf} 1\right),\left(\beta_{1}\right.$ is in $\left.3 \mathrm{mf} 1\right),(z$ is in $4 \mathrm{mf} 3)$, then (PAT efficiency is out1mf6) (1).

7. If ( $b_{2}$ is in1mf1) and ( $\beta_{2}$ is in $\left.2 \mathrm{mf} 1\right),\left(\beta_{1}\right.$ is in $\left.3 \mathrm{mf} 1\right),(z$ is in4mf1), then (PAT efficiency is out1mf7) (1).

8. If $\left(b_{2}\right.$ is in1mf1) and ( $\beta_{2}$ is in $\left.2 \mathrm{mf} 1\right),\left(\beta_{1}\right.$ is in $\left.3 \mathrm{mf} 1\right),(z$ is in $4 \mathrm{mf} 2)$, then (PAT efficiency is out1mf8) (1).

9. If $\left(b_{2}\right.$ is in $\left.1 \mathrm{mf} 1\right)$ and $\left(\beta_{2}\right.$ is in $\left.2 \mathrm{mf} 1\right),\left(\beta_{1}\right.$ is in $\left.3 \mathrm{mf} 1\right),(z$ is in $4 \mathrm{mf} 3)$, then (PAT efficiency is out $\left.1 \mathrm{mf} 9\right)(1)$.

10. If $\left(b_{2}\right.$ is in $\left.1 \mathrm{mf} 1\right)$ and $\left(\beta_{2}\right.$ is in $\left.2 \mathrm{mf} 2\right),\left(\beta_{1}\right.$ is in $\left.3 \mathrm{mf} 1\right),(z$ is in $4 \mathrm{mf} 1)$, then (PAT efficiency is out1mf10) (1). 


\section{References}

1. Seinfeld, J.H.; SNPandis Noone, K. Atmospheric chemistry and physics: From air pollution to climate change. Phys. Today 1998, 51, 88. [CrossRef]

2. Nautiyal, H.; Kumar, V.; Thakur, A. CFD analysis on pumps working as turbines. Hydro Nepal J. Water Energy Environ. 2010, 6, 35-37. [CrossRef]

3. Agarwal, T.J.; Engineering, A. Review of pump as turbine (PAT) for micro-hydropower. Int. J. Emerg. Technol. Adv. Eng. 2012, 2, 163-169.

4. Ramos, H.; Borga, A. Pumps as turbines: An unconventional solution to energy production. Urban Water 1999, 1, 261-263. [CrossRef]

5. Mankbadi, R.R.; Mikhail, S.A. A turbine-pump system for low-head hydropower. Energy Convers. Manag. 1985, 25, 339-344. [CrossRef]

6. Ramos, H.; Covas, D.; Araujo, L.; Mello, M. Available energy assessment in water supply systems. In Proceedings of the 31th International Association for Hydro-Environment Engineering and Research Congress, Seoul, Korea, 11-16 September 2005.

7. Teuteberg, B.H. Design of a Pump-as-Turbine Microhydro System for An Abalone Farm; University of Stellenbosch: Stellenbosch, South Africa, 2010.

8. Yang, S.S.; Kong, F.Y.; Chen, H.; Su, X.H. Effects of blade wrap angle influencing a pump as turbine. J. Fluids Eng. 2012, 134, 061102. [CrossRef]

9. Yang, S.S.; Kong, F.Y.; Shao, F.; Wang, T.; Shen, X.K. Numerical simulation and comparsion of pump and pump as turbine. In ASME 2010 3rd Joint US-European Fluids Engineering Summer Meeting collocated with 8th International Conference on Nanochannels, Microchannels, and Minichannels; American Society of Mechanical Engineers Digital Collection: Montreal, QC, Canada, 2010.

10. Sunsheng, Y.; Fanyu, K.; Hao, C. Effects of blade inlet angle on performance of pump as turbine. J. Cent. South Univ. Sci. Technol. 2013, 44, 108-144.

11. Derakhshan, S.; Mohammadi, B.; Nourbakhsh, A.J. Efficiency improvement of centrifugal reverse pumps. J. Fluids Eng. 2009, 131, 021103. [CrossRef]

12. Smith, K.A.; Gupta, J.N. Neural networks in business: Techniques and applications for the operations researcher. Comput. Oper. Res. 2000, 27, 1023-1044. [CrossRef]

13. Kleijnen, J.P. Kriging metamodeling in simulation: A review. Eur. J. Oper. Res. 2009, 192, 707-716. [CrossRef]

14. Bezerra, M.A.; Santelli, R.E.; Oliveira, E.P.; Villar, L.S.; Escaleira, L.A. Response surface methodology (RSM) as a tool for optimization in analytical chemistry. Talanta 2008, 76, 965-977. [CrossRef] [PubMed]

15. Murata, N.; Yoshizawa, S.; Amari, S.-I. Network information criterion-determining the number of hidden units for an artificial neural network model. IEEE Trans. Neural Netw. 1994, 5, 865-872. [CrossRef] [PubMed]

16. Stathacopoulou, R.; Magoulas, G.D.; Grigoriadou, M. Neural network-based fuzzy modeling of the student in intelligent tutoring systems. In Proceedings of the IJCNN'99, International Joint Conference on Neural Networks, Proceedings (Cat. No.99CH36339), Washington, DC, USA, 10-16 July 1999; IEEE: Piscataway, NJ, USA.

17. Specht, D.F. A general regression neural network. IEEE Trans. Neural Netw. 1991, 2, 568-576. [CrossRef] [PubMed]

18. Gholap, A.; Khan, J.A. Design and multi-objective optimization of heat exchangers for refrigerators. Appl. Energy 2007, 84, 1226-1239. [CrossRef]

19. Ali-Tavoli, M.; Nariman-Zadeh, N.; Khakhali, A.; Mehran, M. Multi-objective optimization of abrasive flow machining processes using polynomial neural networks and genetic algorithms. Mach. Sci. Technol. 2006, 10, 491-510. [CrossRef]

20. Pierret, S.; Coelho, R.F.; Kato, H. Multidisciplinary and multiple operating points shape optimization of three-dimensional compressor blades. Struct. Multidiscip. Optim. 2007, 33, 61-70. [CrossRef]

21. Zhang, J.; Zhu, H.; Yang, C.; Li, Y.; Wei, H. Multi-objective shape optimization of helico-axial multiphase pump impeller based on NSGA-II and ANN. Energy Convers. Manag. 2011, 52, 538-546. [CrossRef]

22. Safikhani, H.; Khalkhali, A.; Farajpoor, M. Pareto based multi-objective optimization of centrifugal pumps using CFD, neural networks and genetic algorithms. Eng. Appl. Comput. Fluid Mech. 2011, 5, 37-48. [CrossRef]

23. Nourbakhsh, A.; Safikhani, H.; Derakhshan, S. The comparison of multi-objective particle swarm optimization and NSGA II algorithm: Applications in centrifugal pumps. Eng. Optim. 2011, 43, 1095-1113. [CrossRef] 
24. Derakhshan, S.; Pourmahdavi, M.; Abdolahnejad, E.; Reihani, A.; Ojaghi, A. Numerical shape optimization of a centrifugal pump impeller using artificial bee colony algorithm. Comput. Fluids 2013, 81, 145-151. [CrossRef]

25. Djavareshkian, M.H.; Latifi, A. Optimization of Wind Turbine Airfoil With Good Stall Characteristics by Genetic Algorithm Using CFD and Neural Network. In ASME 2013 International Mechanical Engineering Congress and Exposition; American Society of Mechanical Engineers Digital Collection: Montreal, QC, Canada, 2013.

26. Giugni, M.; Fontana, N.; Portolano, D. Energy saving policy in water distribution networks. In Proceedings of the International Conference on Renewable Energies and Power Quality (ICREPQ'09), Valencia, Spain, 15-17 April 2009.

27. Pandey, R.; Saini, R.J.E. Performance Analysis of Pump as Turbine Using Artificial Neural Network Techniques. Energy 2008, 22, 24.

28. Shim, H.S.; Kim, K.Y. Numerical investigation on hydrodynamic characteristics of a centrifugal pump with a double volute at off-design conditions. Int. J. Fluid Mach. Syst. 2017, 10, 218-226. [CrossRef]

29. Celik, I.B.; Ghia, U.; Roache, P.J.; Freitas, C.J.; Coleman, H.; Raad, P.E.; Celik, İ.; Freitas, C.; Coleman, H.P. Procedure for estimation and reporting of uncertainty due to discretization in $\{\mathrm{CFD}\}$ applications. J. Fluids Eng. 2008, 130, 78001-78004.

30. Shouqi, Y. Low Specific Speed Centrifugal Pump Theory and Design; Mechanical Industry Press: Beijing, China, 1997.

31. McKay, M.D.; Beckman, R.J.; Conover, W.J. Comparison of three methods for selecting values of input variables in the analysis of output from a computer code. Technometrics 1979, 21, 239-245.

32. Jones, M.; Forero-Hernandez, H.; Zubov, A.; Sarup, B.; Sin, G. Superstructure Optimization of Oleochemical Processes with Surrogate Models, in Computer Aided Chemical Engineering; Elsevier: Amsterdam, The Netherlands, 2018; pp. 277-282.

33. Meireles, M.R.; Almeida, P.E.; Simões, M.G. A comprehensive review for industrial applicability of artificial neural networks. IEEE Trans. Ind. Electron. 2003, 50, 585-601. [CrossRef]

34. Zell, A. Simulation of Neural Networks; Addison-Wesley: Boston, MA, USA, 1994.

35. Rosenblatt, F. The perceptron: A probabilistic model for information storage and organization in the brain. Psychol. Rev. 1958, 65, 386. [CrossRef]

36. Jang, J.S. ANFIS: Adaptive-network-based fuzzy inference system. IEEE Trans. Syst. Man Cybern. 1993, 23, 665-685. [CrossRef]

37. Kodogiannis, V.; Lolis, A. Forecasting financial time series using neural network and fuzzy system-based techniques. Neural Comput. Appl. 2002, 11, 90-102. [CrossRef]

38. Hadi Abdulwahid, A.; Wang, S. A Novel Approach for Microgrid Protection Based upon Combined ANFIS and Hilbert Space-Based Power Setting. Energies 2016, 9, 1042. [CrossRef]

39. Rojas, A.J. Closed-form solution for a class of continuous-time algebraic Riccati equations. In Proceedings of the 48h IEEE Conference on Decision and Control (CDC) held jointly with 2009 28th Chinese Control Conference, Shanghai, China, 15-18 December 2009; IEEE: Piscataway, NJ, USA.

40. Carriere, S.; Caux, S.; Fadel, M.J.N. Synthese LQ pour le contr^ole en vitesse d'un actionneur synchrone autopiloté accouplé directementa une charge mécanique incertaine. Nous 2009, 1, 9.

41. Dchich, K.; Zaafouri, A.; Chaari, A. On Riccati-Genetic Algorithms Approach for Non-convex Problem Resolution. Case of Uncertain Linear System Quadratic Stabilization. Recent Adv. Electrosci. Comput. 2015, 148.

42. Li, S.; Li, K.; Rajamani, R.; Wang, J. Model predictive multi-objective vehicular adaptive cruise control. IEEE Trans. Control Syst. Technol. 2010, 19, 556-566. [CrossRef]

43. Gen, M.; Cheng, R. Genetic Algorithms and Engineering Optimization; John Wiley \& Sons: Hoboken, NJ, USA, 2000; Volume 7.

44. MATLAB. The Language of Technical Computing-Release 14; The MathWorks Inc.: Natick, MA, USA, 2004.

45. Afzal, A.; Kim, K.-Y. Multi-objective optimization of a passive micromixer based on periodic variation of velocity profile. Chem. Eng. Commun. 2015, 202, 322-331. [CrossRef]

46. Kulkarni, K.; Afzal, A.; Kim, K.-Y. Multi-objective optimization of solar air heater with obstacles on absorber plate. Sol. Energy 2015, 114, 364-377. [CrossRef] 
47. Shim, H.-S.; Kim, K.-Y.; Choi, Y.-S. Three-objective optimization of a centrifugal pump to reduce flow recirculation and cavitation. J. Fluids Eng. 2018, 140, 091202. [CrossRef]

48. Pei, J.; Gan, X.; Wang, W.; Yuan, S.; Tang, Y. Multi-objective Shape Optimization on the Inlet Pipe of a Vertical Inline Pump. J. Fluids Eng. 2019, 14, 061108. [CrossRef]

49. Pal, M.; Bharati, P. Introduction to Correlation and Linear Regression Analysis, in Applications of Regression Techniques; Springer: Berlin/Heidelberg, Germany, 2019; pp. 1-18.

50. Sihag, P.; Tiwari, N.; Ranjan, S. Prediction of unsaturated hydraulic conductivity using adaptive neuro-fuzzy inference system (ANFIS). ISH J. Hydraul. Eng. 2019, 25, 132-142. [CrossRef]

(C) 2020 by the authors. Licensee MDPI, Basel, Switzerland. This article is an open access article distributed under the terms and conditions of the Creative Commons Attribution (CC BY) license (http://creativecommons.org/licenses/by/4.0/). 